TRANSACTIONS OF THE

AMERICAN MATHEMATICAL SOCIETY

Volume 356, Number 3, Pages 1155-1183

S 0002-9947(03)03462-7

Article electronically published on October 6, 2003

\title{
CHARACTER DEGREE GRAPHS AND NORMAL SUBGROUPS
}

\author{
I. M. ISAACS
}

\begin{abstract}
We consider the degrees of those irreducible characters of a group $G$ whose kernels do not contain a given normal subgroup $N$. We show that if $N \subseteq G^{\prime}$ and $N$ is not perfect, then the common-divisor graph on this set of integers has at most two connected components. Also, if $N$ is solvable, we obtain bounds on the diameters of the components of this graph and, in the disconnected case, we study the structure of $N$ and of $G$.
\end{abstract}

\section{INTRODUCTION}

Let $G$ be a finite group. As usual, we write $\operatorname{cd}(G)=\{\chi(1) \mid \chi \in \operatorname{Irr}(G)\}$ to denote the set of degrees of the irreducible characters of $G$. A great deal is known about how properties of this set reflect information about the structure of $G$, especially when $G$ is solvable. One way to study the connections between $\operatorname{cd}(G)$ and the normal structure of $G$ was introduced in 4], and that is the approach we take here. The idea of that paper was to focus on the relative degree sets, which, as we will explain, are certain subsets of $\operatorname{cd}(G)$. If $N \triangleleft G$, we define $\operatorname{cd}(G \mid N)=\{\chi(1) \mid \chi \in \operatorname{Irr}(G \mid N)\}$, where $\operatorname{Irr}(G \mid N)=\{\chi \in \operatorname{Irr}(G) \mid N \nsubseteq \operatorname{ker}(\chi)\}$. In other words, the relative degree set $\operatorname{cd}(G \mid N)$ is the set of degrees of those irreducible characters of $G$ whose kernels do not contain $N$.

The study of relative degree sets has proved to be fruitful. Not only does it yield some interesting results about these sets, but significantly, it can also provide a tool for studying the full set $\operatorname{cd}(G)$. For example, in 4] we considered connections between the derived length of a solvable normal subgroup $N \triangleleft G$ and the cardinality of the set $\operatorname{cd}(G \mid N)$. That study led to a much simplified proof of the result of S. Garrison [1], which asserts that if $G$ is solvable and $|\operatorname{cd}(G)|=4$, then the derived length of $G$ is at most 4 .

In this paper, we generalize to the relative case some known results about the character degree graph $\mathcal{G}(G)$ of a finite group. Recall that if $Z$ is an arbitrary set of positive integers, then the common-divisor graph on $Z$ has vertex set $Z$, and distinct vertices $m$ and $n$ are joined if they have a nontrivial common divisor. By definition, the character-degree graph $\mathcal{G}(G)$ of $G$ is the common-divisor graph on the set $\operatorname{cd}(G)$ of irreducible character degrees. Alternatively, we could consider the closely related prime graph on the set $\operatorname{cd}(G)$. (The vertices of the prime graph on a set $Z$ of positive integers are the prime divisors of the members of $Z$, and distinct vertices $p$ and $q$ are joined if $p q$ divides some member of $Z$.) The prime graph and the common divisor graph encode overlapping information about an integer set $Z$,

Received by the editors November 6, 2002.

2000 Mathematics Subject Classification. Primary 20C15.

This research was partially supported by a grant from the U. S. National Security Agency.

(C)2003 American Mathematical Society 
and so for many purposes, the choice of which graph to use is a matter of taste and convenience. Although the literature on this subject seems to refer more often to the prime graph on $\operatorname{cd}(G)$, we prefer the common-divisor graph, and we will use that consistently in this paper.

The degree graph $\mathcal{G}(G)$ always has the "trivial" component, consisting of just the number 1 , and it is reasonable to ask how many other connected components this graph can have. The answer to this question for solvable groups is, perhaps, the oldest known result about degree graphs: If $G$ is solvable, then $\mathcal{G}(G)$ has at most two nontrivial connected components. (What is essentially this theorem was first proved by O. Manz in [7.) Here, we generalize this to the relative degree graph $\mathcal{G}(G \mid N)$, where $N \triangleleft G$. (This is the common divisor graph on the relative degree set $\operatorname{cd}(G \mid N)$.) Note that if $N \subseteq G^{\prime}$, then $1 \notin \operatorname{cd}(G \mid N)$, and so in this case, there is no trivial component.

Theorem A. Let $N \triangleleft G$ and assume that $N^{\prime}<N \subseteq G^{\prime}$. Then $\mathcal{G}(G \mid N)$ has at most two connected components. Furthermore, if the graph is not connected, then all of the degrees in one component are coprime to $\left|N: N^{\prime}\right|$, while all of the degrees in the other component are divisible by every prime divisor of $\left|N: N^{\prime}\right|$.

If $G$ is solvable but not abelian, we can take $N=G^{\prime}$ in Theorem A and we conclude that $\mathcal{G}\left(G \mid G^{\prime}\right)$ has at most two components. Since $\operatorname{cd}\left(G \mid G^{\prime}\right)$ is exactly the set $\operatorname{cd}(G)-\{1\}$, we recover Manz's result.

If $\mathcal{G}(G)$ has two nontrivial components, then a great deal can be said about the structure of $G$. In this situation, for example, the Fitting height of $G$ is at most 4, and in fact, the Fitting height of $G^{\prime}$ is at most 3. (See Theorem 19.6 of $[8]$ and its proof.) But much more is known, and indeed, M. Lewis has completely classified the solvable groups $G$ for which $\mathcal{G}(G)$ has two nontrivial components. Also, both P. Pálfy and J. Zhang have independently obtained detailed information about this case. (See [5, [10] and [12.) Although we are far from a complete classification in the relative case, we do have the following analog of the bound on the Fitting height.

Theorem B. Let $N \triangleleft G$, where $N$ is solvable and $N \subseteq G^{\prime}$. If $\mathcal{G}(G \mid N)$ is not connected, then the Fitting height of $N$ is at most 3. Also, either $N^{\prime \prime}$ is nilpotent or there is an abelian normal subgroup of $G$ that has index 2 in $G^{\prime \prime \prime}$.

It appears likely that $G$ must be solvable in the situation of Theorem B. (To be precise, we are assuming that $N \triangleleft G, N \subseteq G^{\prime}, N$ is solvable and $\mathcal{G}(G \mid N)$ is not connected.) Although we have not been able to prove this in general, we do have the following theorems, which give a great deal of information about this situation. In particular, we see from these results that to prove that $G$ is solvable, it suffices to consider the case where $N$ is a nonabelian $p$-group.

Theorem C. Let $N$ be an abelian p-group, where $N \triangleleft G$ and $N \subseteq G^{\prime}$, and let $P \in \operatorname{Syl}_{p}(G)$. If $\mathcal{G}(G \mid N)$ is not connected, then all of the following occur:

(a) $P \triangleleft G$.

(b) $G / P$ and $P / N$ are abelian.

(c) Each member of $\operatorname{cd}(G \mid N)$ is either a power of $p$ or is coprime to $p$.

(d) $N$ is not central in $P$, but every minimal normal subgroup of $G$ contained in $G^{\prime}$ is central in $G$. 
Theorem D. Let $N$ be solvable, where $N \triangleleft G$ and $N \subseteq G^{\prime}$, and let $p$ be a prime divisor of $\left|N: N^{\prime}\right|$. Assume that $N$ is not a p-group and that $N$ is minimal with the property that $\mathcal{G}(G \mid N)$ is not connected. Then all of the following occur:

(a) $G$ is solvable.

(b) $N$ has a nilpotent normal $p$-complement $M$, and $M$ is abelian if $p=2$.

(c) $N / M$ is abelian except possibly when $p=2$.

(d) $G / M$ has a normal Sylow p-subgroup except possibly when $p=2$.

If $a$ and $b$ are vertices of an arbitrary graph $\mathcal{G}$, we write $d(a, b)$ to denote the distance from $a$ to $b$ in the graph. By definition, $d(a, b)=0$ if $a=b$ and $d(a, b)=\infty$ if $a$ and $b$ are in different components of $\mathcal{G}$. Recall that the diameter of a graph is the maximum distance between two vertices.

A great deal is known about the diameters of the connected components of character-degree graphs. It is comparatively easy, for example, to prove that if $\mathcal{G}(G)$ has two nontrivial components, then each of these components has diameter at most 2, and recently, J. McVey [9] has shown that if $\mathcal{G}(G)$ has just one nontrivial component, then its diameter is at most 3 . It seems reasonable to try to generalize McVey's result by showing under the usual hypotheses, where $N \triangleleft G, N$ is solvable and $N \subseteq G^{\prime}$, that if $\mathcal{G}(G \mid N)$ is connected, then its diameter is at most 3. We can at least prove the following.

Theorem E. Let $N \triangleleft G$, where $N \subseteq G^{\prime}$ and $N$ is solvable. If $\mathcal{G}(G \mid N)$ is not connected, then one component is a complete graph (and so has diameter at most 1 ) and the other component has diameter at most 2 . If the graph is connected, then its diameter is at most 4 and if $N$ is nilpotent, the diameter is at most 3.

\section{Preliminary Results}

In this section, we present some basic facts about the relative degree $\operatorname{set} \operatorname{cd}(G \mid N)$, where $N \triangleleft G$. If $\theta \in \operatorname{Irr}(N)$, we use the standard notation $\operatorname{Irr}(G \mid \theta)$ to denote the set of irreducible characters of $G$ that lie over $\theta$. (These, of course, are just the irreducible constituents of the induced character $\theta^{G}$.) We note that $\operatorname{Irr}(G \mid N)$ is exactly the union of the sets $\operatorname{Irr}(G \mid \theta)$ as $\theta$ runs over all nonprincipal irreducible characters of $N$.

To state our first result, we recall that if $\chi$ is an arbitrary character of a group $X$, then $\operatorname{det}(\chi)$ is the unique linear character of $X$ constructed by taking the determinant of some representation of $X$ that affords $\chi$. We recall also that if $\pi$ is a set of primes and $g$ is an arbitrary element of a finite group, then there is a unique factorization $g=a b$, where $a$ is a $\pi$-element, $b$ is a $\pi^{\prime}$-element and $a$ and $b$ commute. As usual, we refer to $a$ and $b$ as the $\pi$-part and $\pi^{\prime}$-part, respectively, of $g$. In particular, since the set of linear characters of a group $X$ forms a group, we can speak of the $\pi$-part and $\pi^{\prime}$-part of a linear character.

(2.1) Lemma. Let $\theta \in \operatorname{Irr}(N)$, where $N \triangleleft G$, and let $\alpha$ be the $\pi$-part of the linear character $\operatorname{det}(\theta)$. If the set $\operatorname{Irr}(G \mid \theta)$ contains some character $\chi$ having $\pi^{\prime}$-degree, then $\alpha$ is extendible to the stabilizer $G_{\theta}$ of $\theta$ in $G$. In fact, $\alpha$ has an extension to $G_{\theta}$ whose order is a $\pi$-number.

Proof. Let $\eta \in \operatorname{Irr}\left(G_{\theta} \mid \theta\right)$ be the Clifford correspondent of $\chi$ with respect to $\theta$. (See Theorem 6.11 of [3].) Then $\eta^{G}=\chi$, and so $\eta(1)$ divides $\chi(1)$ and $\eta(1)$ is a $\pi^{\prime}$ number. We can thus replace $G$ by $G_{\theta}$ and $\chi$ by $\eta$, and so we can assume that $\theta$ is invariant in $G$. In particular, we have $\chi_{N}=e \theta$, where $e$ is a $\pi^{\prime}$-number. 
Let $\delta=\operatorname{det}(\theta)$ and write $\delta=\alpha \beta$, where $\beta$ is the $\pi^{\prime}$-part of $\delta$. Let $\sigma=\operatorname{det}(\chi)$ and note that $\sigma_{N}=\delta^{e}$. Writing $\sigma=\mu \nu$, where $\mu$ is the $\pi$-part of $\sigma$ and $\nu$ is the $\pi^{\prime}$-part of $\sigma$, we see that $\alpha^{e} \beta^{e}=\delta^{e}=\mu_{N} \nu_{N}$. The orders $o\left(\alpha^{e}\right)$ and $o\left(\beta^{e}\right)$ divide $o(\alpha)$ and $o(\beta)$, and so they are respectively a $\pi$-number and a $\pi^{\prime}$-number, and also, $o\left(\mu_{N}\right)$ and $o\left(\nu_{N}\right)$ divide $o(\mu)$ and $o(\nu)$, and so these too are respectively a $\pi$-number and a $\pi^{\prime}$-number. But the decomposition of $\delta^{e}$ into a $\pi$-part and a $\pi^{\prime}$-part is unique, and therefore $\alpha^{e}=\mu_{N}$.

Now $e$ is a $\pi^{\prime}$-number, and so is coprime to the $\pi$-number $o(\alpha)$. It follows that there exists an integer $f$ so that $e f \equiv 1 \bmod o(\alpha)$, and we have $\left(\mu^{f}\right)_{N}=\alpha^{e f}=\alpha$. Thus $\mu^{f}$ is the required extension of $\alpha$ to $G$.

(2.2) Corollary. Let $N \triangleleft G$ and suppose that $\lambda \in \operatorname{Irr}(N)$ is linear. If $\operatorname{Irr}(G \mid \lambda)$ contains a character whose degree is coprime to o $(\lambda)$, then $\lambda$ is extendible to its stabilizer $G_{\lambda}$ in $G$.

Proof. Apply Lemma 2.1 with $\theta=\lambda$, where $\pi$ is the set of prime divisors of $o(\lambda)$. Then $\lambda$ is the $\pi$-part of $\lambda=\operatorname{det}(\lambda)$, and the result follows.

If $N \triangleleft G$ and $\theta \in \operatorname{Irr}(N)$, we write $\operatorname{cd}(G \mid \theta)=\{\chi(1) \mid \chi \in \operatorname{Irr}(G \mid \theta)\}$.

(2.3) Corollary. Let $\theta \in \operatorname{Irr}(N)$, where $N \triangleleft G$ and $N \subseteq G^{\prime}$. If $\theta$ is nonprincipal, then there is some prime number $p$ that divides every member of $\operatorname{cd}(G \mid \theta)$. In fact, we can choose $p$ so that it divides one of $\theta(1),\left|G: G_{\theta}\right|$ or o $(\operatorname{det}(\theta))$.

Proof. If $\theta$ is nonlinear, we can take $p$ be any divisor of $\theta(1)$ and if $\theta$ is not invariant in $G$, it follows by the Clifford correspondence that we can take $p$ to be any prime divisor of $\left|G: G_{\theta}\right|$. We can assume, therefore, that $\theta$ is linear and that it is invariant in $G$.

Since $\theta$ is linear and nonprincipal, we see that $o(\theta) \neq 1$, and so we can choose $p$ to be a divisor of $o(\theta)$. Let $\lambda$ be the $p$-part of $\operatorname{det}(\theta)=\theta$, so that $\lambda$ is nonprincipal. If some member of $\operatorname{cd}(G \mid \theta)$ fails to be divisible by $p$, then it follows by Lemma 2.1 that $\lambda$ extends to $G_{\theta}=G$. If we let $\mu$ be an extension of $\lambda$ to $G$, then $\lambda=$ $\mu_{N}=1_{N}$, where the second equality holds because $\mu$ is linear and $N \subseteq G^{\prime}$. This is a contradiction, and we conclude that $p$ divides every member of $\operatorname{cd}(G \mid \theta)$, as required.

As an application of what we have done so far, we obtain our first result about the graph $\mathcal{G}(G \mid N)$.

(2.4) Theorem. Let $N \triangleleft G$ with $N \subseteq G^{\prime}$. If $N=U \times V$, where $U$ and $V$ are nontrivial and normal in $G$, then $\mathcal{G}(G \mid N)$ is connected and has diameter at most 3.

Proof. It suffices to show that $d(m, n) \leq 3$, where $m, n \in \operatorname{cd}(G \mid N)$ are arbitrary. Let $\alpha, \beta \in \operatorname{Irr}(N)$ lie under characters in $\operatorname{Irr}(G \mid N)$ with degrees $m$ and $n$, respectively, and note that $\alpha$ and $\beta$ are nonprincipal. Since $N=U \times V$, we can write $\alpha=\alpha_{1} \times \alpha_{2}$ and $\beta=\beta_{1} \times \beta_{2}$, where $\alpha_{1}, \beta_{1} \in \operatorname{Irr}(U)$ and $\alpha_{2}, \beta_{2} \in \operatorname{Irr}(V)$.

Suppose first that $\alpha_{1}$ and $\beta_{1}$ are both nonprincipal. Let $\tau \in \operatorname{Irr}(V)$ be nonprincipal and choose $s \in \operatorname{cd}\left(G \mid\left(\alpha_{1} \times \tau\right)\right)$ and $t \in \operatorname{cd}\left(G \mid\left(\beta_{1} \times \tau\right)\right)$. Then $m, s \in \operatorname{cd}\left(G \mid \alpha_{1}\right)$, and so $m$ and $s$ have a nontrivial common divisor by Corollary 2.3, and hence they are either equal or joined in $\mathcal{G}(G \mid N)$. Similarly, $n, t \in \operatorname{cd}\left(G \mid \beta_{1}\right)$, and so $n$ and $t$ are equal or joined in $\mathcal{G}(G \mid N)$. Also $s, t \in \operatorname{cd}(G \mid \tau)$, and so $s$ and $t$ are either equal or joined in $\mathcal{G}(G \mid N)$. We see that in this case, the distance $d(m, n) \leq 3$, as required. 
We can now suppose that one of $\alpha_{1}$ or $\beta_{1}$ is principal, and similarly, we can suppose that one of $\alpha_{2}$ or $\beta_{2}$ is principal. By symmetry, we can assume that $\alpha_{1}=1_{U}$, and thus since $\alpha=\alpha_{1} \times \alpha_{2}$ is nonprincipal, we see that $\alpha_{2}$ is nonprincipal, and thus $\beta_{2}=1_{V}$. Finally, since $\beta=\beta_{1} \times \beta_{2}$ is nonprincipal, we see that $\beta_{1}$ must be nonprincipal.

Now choose $s \in \operatorname{cd}\left(G \mid\left(\beta_{1} \times \alpha_{2}\right)\right)$. Then $m, s \in \operatorname{cd}\left(G \mid \alpha_{2}\right)$ and $n, s \in \operatorname{cd}\left(G \mid \beta_{1}\right)$, and since $\beta_{1}$ and $\alpha_{2}$ are nonprincipal, we see by Corollary 2.3 that $m$ and $s$ have a nontrivial common divisor and that $n$ and $s$ also have a nontrivial common divisor. It follows in this case that $d(m, n) \leq 2$, and the proof is complete.

(2.5) Corollary. Let $N \triangleleft G$, where $N$ is nilpotent and $N \subseteq G^{\prime}$. If $N$ is not a p-group, then $\mathcal{G}(G \mid N)$ is connected and has diameter at most 3 .

Perhaps it is worth noting that if $N$ is a $p$-group in the situation of Corollary 2.5, then $\mathcal{G}(G \mid N)$ need not be connected. In fact, examples exist for all primes $p$. To see this, let $N$ be extra-special of order $p^{3}$, where $N$ has exponent $p$ if $p>2$ and $N \cong Q_{8}$ if $p=2$. Let $C$ be a cyclic group of prime order $q \neq p$ that acts on $N$ in such a way that $\mathbf{C}_{N}(C)=\mathbf{Z}(N)$ and let $G=N C$ be the semidirect product. (Note that we can take $q$ to be any prime divisor of $p-1$ or $p+1$.) It is not hard to see in this situation that $\operatorname{cd}(G \mid N)=\{p, q\}$, and thus $\mathcal{G}(G \mid N)$ has two connected components.

We also have the following result, which is quite similar to Theorem 2.4.

(2.6) Theorem. Let $N \triangleleft G$ with $N \subseteq G^{\prime}$ and suppose that $K \triangleleft G$ and $K \cap N=1$. If $K \cap G^{\prime}>1$, then $\mathcal{G}(G \mid N)$ is connected and has diameter at most 3 .

Proof. As in the proof of Theorem 2.4, we show that $d(m, n) \leq 3$, where $m, n \in$ $\operatorname{cd}(G \mid N)$ are arbitrary. Replacing $K$ by $K \cap G^{\prime}$, we can assume that $1<K \subseteq G^{\prime}$ and we let $\tau \in \operatorname{Irr}(K)$ be nonprincipal. Choose $\alpha, \beta \in \operatorname{Irr}(N)$ lying under characters in $\operatorname{Irr}(G \mid N)$ with degrees $m$ and $n$, respectively, and let $s \in \operatorname{cd}(G \mid(\alpha \times \tau))$ and $t \in \operatorname{cd}(G \mid(\beta \times \tau))$. Since $\alpha$ and $\beta$ are nonprincipal, we see that $s$ and $t$ lie in $\operatorname{cd}(G \mid N)$.

Now $m, s \in \operatorname{cd}(G \mid \alpha)$ and $n, t \in \operatorname{cd}(G \mid \beta)$, and thus $d(m, s) \leq 1$ and $d(t, n) \leq 1$ by Corollary 2.3. We can also apply Corollary 2.3 to $s, t \in \operatorname{Irr}(G \mid \tau)$, and the result follows.

(2.7) Corollary. Let $N \triangleleft G$ with $N \subseteq G^{\prime}$. If $\mathcal{G}(G \mid N)$ is not connected, then $N$ contains every minimal normal subgroup of $G$ that is contained in $G^{\prime}$.

\section{ORBIT SIZES}

Let $G$ be an arbitrary finite group, and suppose that $G$ acts on a finite abelian group $A$. In certain situations, we can obtain some useful information about the sizes of the $G$-orbits on $A$. We present part of a theorem of T. Yuster [11] and then a related lemma that applies under weaker hypotheses. (We have included a proof of Yuster's result for completeness and for comparison with the proof of our second lemma.) In the following, we will write $A$ additively and for $x \in A$, we write $x^{G}$ to denote the $G$-orbit containing $x$.

(3.1) Lemma. Suppose that $G$ acts on an abelian group $A$ and let $x, y \in A$ be elements such that the orbit sizes $\left|x^{G}\right|$ and $\left|y^{G}\right|$ are coprime. If $|G|$ and $|A|$ are coprime, then $\left|(x+y)^{G}\right|=\left|x^{G}\right|\left|y^{G}\right|$. 
Proof. We have $\left|G: G_{x}\right|=\left|x^{G}\right|$ and $\left|G: G_{y}\right|=\left|y^{G}\right|$, and since the indices of the subgroups $G_{x}$ and $G_{y}$ in $G$ are coprime, we conclude that $G=G_{x} G_{y}$. It follows that $\left|G: G_{x} \cap G_{y}\right|=\left|G: G_{x}\right|\left|G: G_{y}\right|=\left|x^{G}\right|\left|y^{G}\right|$. Also, writing $z=x+y$, it is clear that $G_{x} \cap G_{y} \subseteq G_{z}$, and so to prove the result, it suffices to establish the reverse containment.

Let $U=\left\langle x^{G}\right\rangle$ and $V=\left\langle y^{G}\right\rangle$ and write $W=V \cap U$. Then $U, V$ and $W$ are $G$-invariant subgroups of $A$, and $G$ permutes the cosets of $W$ in $A$. Let $L$ be the stabilizer in $G$ of the coset $W+x$ and note that $L \supseteq G_{x}$. Also, $L$ stabilizes the group $B=\langle W, x\rangle$ and $L$ acts trivially on $B / W$, and hence $[B, L] \subseteq W$. Also, because $|L|$ and $|A|$ are coprime, it follows from Fitting's lemma that $B=\mathbf{C}_{B}(L)+[B, L]$, and thus we can write $x=c+w$, where $L$ fixes $c$ and $w \in[B, L] \subseteq W$.

Now define the map $T: A \rightarrow A$ by $T(a)=\sum a^{t}$, where the sum runs over $t \in G_{x}$, and observe that $T$ is a homomorphism. Recall that $G_{x} G_{y}=G$, and so $G_{x}$ is transitive on the orbit $y^{G}$. If $a \in y^{G}$, it follows that $T(a)$ is a multiple of the orbit sum, and thus $T$ maps the set $y^{G}$ into $\mathbf{C}_{A}(G)$. We conclude that $T$ maps $V=\left\langle y^{G}\right\rangle$ into $\mathbf{C}_{A}(G)$, and so in particular, $T(w)$ is fixed by $G$.

Now $T(x)=\left|G_{x}\right| x$ and also, since $c$ is $L$-fixed and $G_{x} \subseteq L$, we have $T(c)=\left|G_{x}\right| c$. We thus have $\left|G_{x}\right| w=\left|G_{x}\right|(x-c)=T(x)-T(c)=T(w) \in \mathbf{C}_{A}(G)$. But $\left|G_{x}\right|$ and $|A|$ are coprime, and it follows that $w \in \mathbf{C}_{A}(G)$. Since $G$ fixes $w$ and $L$ fixes $c$, we see that $x=c+w$ is fixed by $L$, and so $L \subseteq G_{x}$.

Since $x \in U$, we see that $W+x \subseteq U \cap(V+x)$, and in fact, equality holds here. To see this, note that if $u \in U \cap(V+x)$, we can write $u=v+x$, with $v \in V$. Then $v=u-x \in U$, and thus $v \in U \cap V=W$ and $u=v+x \in W+x$, as wanted. Since $y \in V$, we see that $z=x+y \in V+x$, and thus $G_{z}$ stabilizes the coset $V+x$. Of course, $G_{z}$ also stabilizes the $G$-invariant subgroup $U$, and thus $G_{z}$ stabilizes $(V+x) \cap U=W+x$, and we conclude that $G_{z} \subseteq L$.

We now have $G_{z} \subseteq L \subseteq G_{x}$ and similarly, $G_{z} \subseteq G_{y}$. Thus $G_{z} \subseteq G_{x} \cap G_{y}$, and this completes the proof.

If we drop the assumption that $|G|$ and $|A|$ are coprime in Lemma 3.1, then the result becomes false. But a weaker assumption is sufficient to obtain the weaker conclusion that if $\left|x^{G}\right|>1$, then $\left|x^{G}\right|$ and $\left|(x+y)^{G}\right|$ are not coprime.

(3.2) Lemma. Suppose that $G$ acts on an abelian group $A$ and let $x, y \in A$ be elements such that the orbit sizes $\left|x^{G}\right|$ and $\left|y^{G}\right|$ are coprime. Suppose that there exists a subgroup $K \subseteq G_{x}$ such that $|K|$ and $|A|$ are coprime and $G_{x}=K\left(G_{x} \cap G_{y}\right)$. Then $\left|x^{G}\right|$ and $\left|(x+y)^{G}\right|$ are not coprime except in the trivial case where $\left|x^{G}\right|=1$.

Proof. Write $z=x+y$ and assume that the orbit sizes $\left|x^{G}\right|$ and $\left|z^{G}\right|$ are coprime. We work to show that $x$ is invariant in $G$. Now $\left|G: G_{x}\right|=\left|x^{G}\right|$ and $\left|G: G_{y}\right|=\left|y^{G}\right|$ are coprime, and thus $G=G_{x} G_{y}=K\left(G_{x} \cap G_{y}\right) G_{y}=K G_{y}$, and it follows that $K$ is transitive on $y^{G}$. Also, since we are assuming that $\left|x^{G}\right|$ and $\left|z^{G}\right|$ are coprime and we have $G_{x} \cap G_{y} \subseteq G_{z}$, we see that $G=G_{x} G_{z}=K\left(G_{x} \cap G_{y}\right) G_{z}=K G_{z}$, and thus $K$ is transitive on $z^{G}$.

Now define $T: A \rightarrow A$ by $T(a)=\sum a^{k}$, where $k$ runs over $K$, and note that $T(y)$ and $T(z)$ are $G$-invariant since $K$ is transitive on the $G$-orbits of $y$ and $z$. Since $K \subseteq G_{x}$, we have $|K| x=T(x)=T(z)-T(y) \in \mathbf{C}_{A}(G)$, and thus $x \in \mathbf{C}_{A}(G)$ as required, since $|K|$ and $|A|$ are coprime. 
When are the hypotheses of Lemma 3.2 satisfied? Suppose that we are in the situation where $G$ acts on the abelian group $A$ and we have elements $x, y \in A$ in $G$ orbits of coprime size. In this situation, we know that if a subgroup $K \subseteq G_{x}$ exists such that $G_{x}=K\left(G_{x} \cap G_{y}\right)$, then $K$ is transitive on $y^{G}$, and thus $\left|y^{G}\right| \overline{\text { divides }}|K|$. If we want $|K|$ and $|A|$ to be coprime, therefore, it is necessary that $\left|y^{G}\right|$ and $|A|$ are coprime. Conversely, if $\left|y^{G}\right|$ and $|A|$ are coprime, let $\pi$ be the set of prime divisors of the orbit size $\left|y^{G}\right|$. If $G_{x}$ has a Hall $\pi$-subgroup $K$, then $K\left(G_{x} \cap G_{y}\right)=G_{x}$, and the hypotheses of Lemma 3.2 will be satisfied. We see, therefore, that to take advantage of Lemma 3.2, it is useful to have conditions (such as solvability) that are sufficient to guarantee the existence of a Hall $\pi$-subgroup.

(3.3) Corollary. Suppose that $G$ acts on an abelian group $A$. Let $x, y \in A$ be elements such that the orbit sizes $\left|x^{G}\right|$ and $\left|y^{G}\right|$ are coprime and assume also that $\left|y^{G}\right|$ and $|A|$ are coprime. If $G_{x}$ has a Hall $\pi$-subgroup, where $\pi$ is the set of prime divisors of $\left|y^{G}\right|$, then $\left|x^{G}\right|$ and $\left|(x+y)^{G}\right|$ are not coprime except in the trivial case where $\left|x^{G}\right|=1$.

The following is an application of Corollary 3.3 to the study of the relative degree graph $\mathcal{G}(G \mid N)$.

(3.4) Theorem. Let $N \triangleleft G$ with $N \subseteq G^{\prime}$ and suppose that $m, n \in \operatorname{cd}(G \mid N)$ are coprime to $|N|$. Then $m$ and $n$ have distance at most 2 in $\mathcal{G}(G \mid N)$, and in particular, they lie in the same connected component of this graph.

If we assume that $G / N$ is solvable, then the proof of Theorem 3.4 becomes quite elementary because of the guaranteed existence of Hall subgroups. In order to prove the general case however, we need the following lemma, whose proof is an easy application of the Ito-Michler theorem. Unfortunately, however, the proof of the Ito-Michler theorem relies on the classification of simple groups.

(3.5) Lemma. Let $\alpha \in \operatorname{Irr}(N)$, where $N \triangleleft G$, and assume that $\alpha$ extends to its stabilizer $G_{\alpha}$. Let $\pi$ be a set of primes and suppose that for every character $\chi \in \operatorname{Irr}(G \mid \alpha)$, the quotient $\chi(1) / \alpha(1)$ is a $\pi$-number. Then $G / N$ has an abelian Hall $\pi^{\prime}$-subgroup $A / N$ with $A \triangleleft G_{\alpha}$.

Proof. Let $\hat{\alpha} \in \operatorname{Irr}\left(G_{\alpha}\right)$ be an extension of $\alpha$ and let $\theta \in \operatorname{Irr}\left(G_{\alpha} / N\right)$ be arbitrary. By Gallagher's theorem, $\hat{\alpha} \theta \in \operatorname{Irr}\left(G_{\alpha} \mid \alpha\right)$, and thus by the Clifford correspondence $\chi=(\hat{\alpha} \theta)^{G}$ lies in $\operatorname{Irr}(G \mid \alpha)$. By hypothesis, therefore, $\theta(1)\left|G: G_{\alpha}\right|=\chi(1) / \alpha(1)$ is a $\pi$-number, and thus $\left|G: G_{\alpha}\right|$ is a $\pi$-number and $\theta(1)$ is a $\pi$-number for every choice of $\theta$.

If $q$ is a prime that is not in $\pi$, then no irreducible character of $G_{\alpha} / N$ has degree divisible by $q$, and in this situation, the Ito-Michler theorem guarantees that $G_{\alpha} / N$ has a normal abelian Sylow $q$-subgroup. It follows that $G_{\alpha} / N$ has a normal abelian Hall $\pi^{\prime}$-subgroup $A / N$. Finally, since $\left|G: G_{\alpha}\right|$ is a $\pi$-number, we see that $A / N$ is actually a Hall $\pi^{\prime}$-subgroup of $G / N$, and the proof is complete.

Proof of Theorem 3.4. We can assume that $m$ and $n$ are coprime, and we seek a degree in $\operatorname{cd}(G \mid N)$ that is coprime to neither $m$ nor $n$. Let $\alpha, \beta \in \operatorname{Irr}(N)$ lie under characters in $\operatorname{Irr}(G \mid N)$ with degrees $m$ and $n$, respectively, and note that neither $\alpha$ nor $\beta$ is principal. Also $\alpha(1)$ divides both $m$ and $|N|$, and since these numbers are coprime, we see that $\alpha(1)=1$. Furthermore, the order of $\alpha$ divides $|N|$, which is coprime to $m$, and thus by Corollary 2.2 , we see that $\alpha$ extends to its stabilizer 
$G_{\alpha}$. But $N \subseteq G^{\prime}$ and $\alpha$ is linear, and so $\alpha$ does not extend to $G$ and we conclude that $G_{\alpha}<G$. Similarly, $\beta$ extends to $G_{\beta}<G$.

Write $a=\left|G: G_{\alpha}\right|$ and $b=\left|G: G_{\beta}\right|$. Then $a>1$ and $b>1$ and also, since $a$ divides $m$ and $b$ divides $n$, we see that $a$ and $b$ are coprime and that each of them is coprime to $|N|$. Now let $\pi$ be the set of prime divisors of the members of $\operatorname{cd}(G \mid \alpha)$. If $n$ is not a $\pi^{\prime}$-number, then there exists a prime $q$ dividing $n$ and also dividing some number $k \in \operatorname{cd}(G \mid \alpha)$. Then $k$ and $n$ are both divisible by $q$ and also $k$ and $m$ are both divisible by $a$, and so we are done in this case. We can thus assume that $n$ is a $\pi^{\prime}$-number, and thus $b$ is a $\pi^{\prime}$-number. Similarly, we can assume that $a$ is not divisible by any prime divisor of a member of $\operatorname{cd}(G \mid \beta)$.

By Lemma 3.5, we see that $G_{\alpha} / N$ has an abelian Hall $\pi^{\prime}$-subgroup, and thus it has a Hall subgroup for the set of prime divisors of $b$ and similarly, $G_{\beta} / N$ has a Hall subgroup for the set of prime divisors of $a$.

The group $G / N$ acts on the abelian group $A$ of linear characters of $N$, and we see that $a$ and $b$ are the sizes of the orbits of $\alpha$ and $\beta$, respectively. Now $a>1$, $b>1$ and $a$ and $b$ are coprime to each other and to $|A|$. Also, the appropriate Hall subgroups exist, and hence it follows from Corollary 3.3 that $\left|G: G_{\alpha \beta}\right|$ is not coprime to $a$ or to $b$, and in particular, $\alpha \beta$ is not the principal character. If $k \in \operatorname{cd}(G \mid \alpha \beta)$, therefore, then $k \in \operatorname{cd}(G \mid N)$ and $k$ is not coprime to either $m$ or $n$. This completes the the proof.

\section{Two COMPONENTS}

We are now ready to begin work toward proving Theorem A. The following result is crucial.

(4.1) Theorem. Let $M \subseteq N \subseteq G^{\prime}$, where $M, N \triangleleft G$ and $N / M$ is a nontrivial p-group. Then all $p^{\prime}$-numbers in $\operatorname{cd}(G \mid M)$ lie in the same connected component of $\mathcal{G}(G \mid M)$ and the distance between any two of them is at most 3. Furthermore, if $m$ is a $p^{\prime}$-number in $\operatorname{cd}(G \mid M)$ and $k \in \operatorname{cd}((G / M) \mid(N / M))$ is arbitrary, then the distance from $m$ to $k$ in $\mathcal{G}(G \mid N)$ is at most 2 .

Proof. There is nothing to prove unless $\operatorname{cd}(G \mid M)$ contains a $p^{\prime}$-number, and so we consider a nonprincipal character $\alpha \in \operatorname{Irr}(M)$ such that $\operatorname{cd}(G \mid \alpha)$ contains a $p^{\prime}$ number. Let $\varphi \in \operatorname{Irr}(N / M)$ be nonprincipal and write $u=\left|G: G_{\varphi}\right|$. We claim that some member of $\operatorname{cd}(G \mid \alpha)$ is a multiple of $u$. Also, if $u=1$, we claim that some member of $\operatorname{cd}(G \mid \alpha)$ is a multiple of $p$ and that every member of $\operatorname{cd}(G \mid \varphi)$ is a multiple of $p$. Assuming all of this for the moment, we complete the proof of the Theorem.

Let $m, n \in \operatorname{cd}(G \mid M)$ be $p^{\prime}$-numbers and let $k \in \operatorname{cd}((G / M) \mid(N / M))$. Choose $\alpha, \beta \in \operatorname{Irr}(M)$ lying under characters in $\operatorname{Irr}(G \mid M)$ of degree $m$ and $n$, respectively, and choose $\varphi \in \operatorname{Irr}(N / M)$ lying under a character in $\operatorname{Irr}((G / M) \mid(N / M))$ of degree $k$. Let $u=\left|G: G_{\varphi}\right|$ and note that $\alpha, \beta$ and $\varphi$ are nonprincipal. By the assertions of the first paragraph, there exist $s \in \operatorname{cd}(G \mid \alpha)$ and $t \in \operatorname{cd}(G \mid \beta)$ such that both $s$ and $t$ are multiples of $u$, and if $u=1$, we can choose $s$ and $t$ so that both are multiples of $p$. In either case, therefore, $s$ and $t$ are not coprime. Also, $m$ and $s$ lie in $\operatorname{cd}(G \mid \alpha)$, and so they are not coprime by Corollary 2.3 , and similarly, $n$ and $t$ are not coprime. It follows that $d(m, n) \leq 3$ in $\mathcal{G}(G \mid M)$, as desired. Also, we see that $u$ divides $k$, and by the assertion of the first paragraph, if $u=1$, then $p$ divides $k$. It follows that $k$ and $s$ are not coprime, and thus $d(m, k) \leq 2$ in $\mathcal{G}(G \mid N)$. 
What remains now is to prove the assertions of the first paragraph. We are assuming that we are given $\alpha \in \operatorname{Irr}(M)$, where $\alpha$ is nonprincipal, and that there exists $\chi \in \operatorname{Irr}(G \mid \alpha)$ such that $\chi(1)$ is a $p^{\prime}$-number. We are also given a nonprincipal character $\varphi \in \operatorname{Irr}(N / M)$, where $\left|G: G_{\varphi}\right|=u$, and our first task is to produce a character $\eta \in \operatorname{Irr}(G \mid \alpha)$ such that $u$ divides $\eta(1)$.

Let $\psi$ be an irreducible constituent of $\chi_{N}$ such that $\psi$ lies over $\alpha$. Then $\psi(1)$ divides the $p^{\prime}$-number $\chi(1)$, and hence $\psi(1)$ is coprime to $|N: M|$, which is a power of $p$. It follows that $\psi_{M}$ is irreducible, and so $\psi_{M}=\alpha$ and $\alpha$ extends to $N$, and in particular, $N \subseteq G_{\alpha}$. Now let $\lambda$ be the $p$-part of $\operatorname{det}(\alpha)$ and note that since $\chi(1)$ is a $p^{\prime}$-number, Lemma 2.1 guarantees that $\lambda$ has an extension to $G_{\alpha}$. The restriction of this extension to $N$ is thus a $G_{\alpha}$-invariant extension of $\lambda$ to $N$.

We show next that the $p^{\prime}$-part $\mu$ of $\operatorname{det}(\alpha)$ also has a $G_{\alpha}$-invariant extension to $N$. Let $\nu$ be the $p^{\prime}$-part of $\operatorname{det}(\psi)$ and note that since $(\operatorname{det}(\psi))_{N}=\operatorname{det}(\alpha)$, we have $\nu_{M}=\mu$. In fact, $\nu$ is the unique extension of $\mu$ to $N$ that has $p^{\prime}$-order. (This follows easily from the fact that restriction defines a homomorphism from the abelian group of linear characters of $N$ into the group of linear characters of $M$, and the kernel of this homomorphism is a $p$-group since it is exactly the group of linear characters of $N / M$.) Now $\alpha$ uniquely determines $\mu$ and $\mu$ uniquely determines $\nu$, and it follows that $\nu$ is $G_{\alpha}$-invariant, as wanted.

We have now seen that both the $p$-part and the $p^{\prime}$-part of $\operatorname{det}(\alpha)$ have $G_{\alpha^{-}}$ invariant extensions to $N$, and it follows that $\operatorname{det}(\alpha)$, which is their product, also has a $G_{\alpha}$-invariant extension $\tau \in \operatorname{Irr}(N)$. We know that $\alpha$ extends to $N$ and that $\alpha(1)$ is coprime to $|N: M|$, and it follows that $\alpha$ has a unique extension $\gamma \in \operatorname{Irr}(N)$ such that $\operatorname{det}(\gamma)=\tau$. (This is exactly Lemma 6.24 of [3].) Since $\gamma$ is uniquely determined by $\alpha$ and $\tau$ and each of these characters is $G_{\alpha}$-invariant, it follows that $G_{\alpha} \subseteq G_{\gamma}$. (In fact, since $\gamma_{N}=\alpha$, the reverse containment is clear, but we shall not need this observation.)

Now $\varphi \in \operatorname{Irr}(N / M)$ and $\gamma_{M}=\alpha \in \operatorname{Irr}(M)$, and hence $\varphi \gamma$ is irreducible by Gallagher's theorem. (See Corollary 6.17 of [3].) Furthermore, $(\varphi \gamma)_{M}$ is a multiple of $\alpha$, and hence $G_{\varphi \gamma} \subseteq G_{\alpha} \subseteq G_{\gamma}$. But Gallagher's theorem tells us that $\gamma$ and $\varphi \gamma$ uniquely determine $\varphi$, and it follows that $G_{\varphi \gamma} \subseteq G_{\varphi}$, and thus $u$ divides $\left|G: G_{\varphi \gamma}\right|$. If $\xi \in \operatorname{Irr}(G \mid \varphi \gamma)$, therefore, we see that $u$ divides $\xi(1)$ and $\xi \in \operatorname{Irr}(G \mid \alpha)$, as wanted.

Next, we assume that $u=1$, so that $\varphi$ is $G$-invariant. If $\operatorname{cd}(G \mid \varphi)$ contains a $p^{\prime}$-number, then $\varphi(1)$ must be a $p^{\prime}$-number, and hence $\varphi(1)=1$ since $N / M$ is a $p$-group. Also, the order of the linear character $\varphi$ is a $p$-power, and so $\varphi$ extends to $G_{\varphi}=G$ by Corollary 2.2. But this is impossible since $N \subseteq G^{\prime}$ and $\varphi$ is linear and nonprincipal. This contradiction shows that every member of $\operatorname{cd}(G \mid \varphi)$ is divisible by $p$.

To complete the proof, we must show that $p$ divides some member of $\operatorname{cd}(G \mid \alpha)$. It suffices, therefore, to show that either $p$ divides some member of $\operatorname{cd}(G \mid \gamma)$ or some member of $\operatorname{cd}(G \mid \varphi \gamma)$. We assume that this is false and work to obtain a contradiction. Since $\varphi(1)$ divides each degree in $\operatorname{cd}(G \mid \varphi \gamma)$, we see that $p$ does not divide $\varphi(1)$, and thus $\varphi$ is linear. Also, we know that $\varphi$ is $G$-invariant, and it follows that $G_{\gamma}=G_{\varphi \gamma}$, and this common stabilizer has $p^{\prime}$-index. Also, by Lemma 2.1, we know that the $p$-part of $\operatorname{det}(\gamma)$ and the $p$-part of $\operatorname{det}(\varphi \gamma)$ both extend to $G_{\gamma}$. But $\operatorname{det}(\varphi \gamma)=\operatorname{det}(\gamma) \varphi^{\gamma(1)}$, and we see that the quotient of the two determinants is $\varphi^{\gamma(1)}$, which is a linear character of $p$-power order. It follows, therefore, that $\varphi^{\gamma(1)}$ is also the quotient of the $p$-parts of these determinants, and hence it is extendible 
to $G_{\gamma}$. Since $\gamma(1)$ is not divisible by $p$ and $\varphi$ has $p$-power order, we see that $\varphi$ is a power of $\varphi^{\gamma(1)}$, and it follows that $\varphi$ extends to some linear character $\sigma$ of $G_{\gamma}$. Then $\sigma^{G}(1)=\left|G: G_{\gamma}\right|$ is not divisible by $p$, and thus some irreducible constituent $\xi$ of $\sigma^{G}$ also has degree not divisible by $p$. But $\xi \in \operatorname{Irr}(G \mid \varphi)$, and this contradicts the result of the previous paragraph.

(4.2) Corollary. Let $N \triangleleft G$ with $N \subseteq G^{\prime}$, and suppose that $N / M$ is a nontrivial p-group, where $M \triangleleft G$. If $m, n \in \operatorname{cd}(G \mid N)$ are $p^{\prime}$-numbers, then $d(m, n) \leq 3$ in $\mathcal{G}(G \mid N)$. Furthermore, if $d(m, n)=3$, then $m$ and $n$ lie in $\operatorname{cd}(G \mid M)$ and their distance in $\mathcal{G}(G \mid M)$ is also 3 .

Proof. If $m, n \in \operatorname{cd}(G \mid M)$, then by Theorem 4.1, the distance between $m$ and $n$ in the subgraph $\mathcal{G}(G \mid M)$ of $\mathcal{G}(G \mid N)$ is at most 3, and there is nothing further to prove. We can assume, then, that $m \notin \operatorname{cd}(G \mid M)$, and thus $m \in \operatorname{cd}((G / M) \mid(N / M))$. If $n \in \operatorname{cd}(G \mid M)$, then by Theorem 4.1, we have $d(m, n) \leq 2$ in $\mathcal{G}(G \mid N)$, and we are done in this case, too. Finally, we can assume that both $m$ and $n$ lie in $\operatorname{cd}((G / M) \mid(N / M))$, and we can apply Theorem 3.4 in the group $G / M$ with respect to the $p$-subgroup $N / M$. We see from that result that the distance between $m$ and $n$ in the subgraph $\mathcal{G}((G / M) \mid(N / M))$ of $\mathcal{G}(G \mid N)$ is at most 2, and the proof is complete.

The following result includes most of Theorem A.

(4.3) Corollary. Let $N \triangleleft G$ with $N \subseteq G^{\prime}$. Suppose that the prime number $p$ divides $\left|N: N^{\prime}\right|$ and that $\mathcal{G}(G \mid N)$ is not connected. Then $\mathcal{G}(G \mid N)$ has exactly two components: the multiples of $p$ in $\operatorname{cd}(G \mid N)$ and the $p^{\prime}$-numbers in $\operatorname{cd}(G \mid N)$. The latter component, furthermore, has diameter at most 3.

Proof. Let $X$ be the set of $p^{\prime}$-numbers in $\operatorname{cd}(G \mid N)$. Since $p$ divides $\left|N: N^{\prime}\right|$, there exists $M \triangleleft G$ such that $M<N$ and $N / M$ is a $p$-group, and thus Corollary 4.2 applies and we conclude that $X$ is contained in a single component of $\mathcal{G}(G \mid N)$. Since this graph is not connected, $X$ must be a proper subset of $\operatorname{cd}(G \mid N)$, and we denote the complement by $Y$. But $Y$ consists of multiples of $p$, and thus $Y$ is contained in a single component of $\mathcal{G}(G \mid N)$, and hence $Y$ is proper in $\operatorname{cd}(G \mid N)$. It follows that $X$ and $Y$ are both nonempty and that they are exactly the components of $\mathcal{G}(G \mid N)$, as required. Furthermore, if $m, n \in X$, then we know by Corollary 4.2 that the distance between $m$ and $n$ in $\mathcal{G}(G \mid N)$ is at most 3, and thus their distance in the component $X$ is at most 3. It follows that $X$ has diameter at most 3 .

Proof of Theorem A. All that remains to be shown is that if $\mathcal{G}(G \mid N)$ has two components $X$ and $Y$, then all of the prime divisors of $\left|N: N^{\prime}\right|$ divide the numbers in the same component. If this is false, then we can assume that there are primes $p$ and $q$ dividing $\left|N: N^{\prime}\right|$ such that every member of $X$ is divisible by $q$ but not by $p$ and every member of $Y$ is divisible by $p$ but not by $q$.

Since $N / N^{\prime}$ is an abelian group with order divisible by more than one prime, we see that this group is a direct product of nonidentity characteristic subgroups, and thus by Theorem 2.4, the graph $\mathcal{G}\left(\left(G / N^{\prime}\right) \mid\left(N / N^{\prime}\right)\right)$ is connected. Since this is a subgraph of $\mathcal{G}(G \mid N)$, it must be contained in one of the two components of the larger graph, and so we can assume that $\operatorname{cd}\left(\left(G / N^{\prime}\right) \mid\left(N / N^{\prime}\right)\right) \subseteq Y$.

Now let $N / M$ be a nontrivial abelian $p$-group, where $M \triangleleft G$. Since $M \supseteq N^{\prime}$, we see that $\operatorname{cd}((G / M) \mid(N / M)) \subseteq \operatorname{cd}\left(\left(G / N^{\prime}\right) \mid\left(N / N^{\prime}\right)\right) \subseteq Y$. If we choose $m \in X$, 
therefore, we have $m \notin \operatorname{cd}((G / M) \mid(N / M))$, and thus $m \in \operatorname{cd}(G \mid M)$. But since $m \in X$, we know that $m$ is a $p^{\prime}$-number, and it follows from Theorem 4.1 that $m$ is at a distance at most 2 from an arbitrary member $k \in \operatorname{cd}((G / M) \mid(N / M))$. But $m \in X$ and $k \in Y$, and this is a contradiction.

If we assume in Corollary 4.3 that $N$ is solvable, we can get better control of the diameter of the component consisting of $p^{\prime}$-numbers. (Of course, the component consisting of multiples of $p$ is a complete graph with diameter at most 1.) The following is the part of Theorem E that applies to the situation where $\mathcal{G}(G \mid N)$ is not connected.

(4.4) Corollary. Let $N \triangleleft G$, where $N \subseteq G^{\prime}$ and $N$ is solvable, and assume that $\mathcal{G}(G \mid N)$ is not connected. Then the component consisting of numbers coprime to $\left|N: N^{\prime}\right|$ has diameter at most 2 .

Proof. We work by induction on $|N|$. Let $M<N$, where $M \triangleleft G$ and $N / M$ is a $p$-group, and suppose that $m, n \in \operatorname{cd}(G \mid N)$ are $p^{\prime}$-numbers. We need to show that $d(m, n) \leq 2$ in $\mathcal{G}(G \mid N)$. By Corollary 4.2 , we know that $d(m, n) \leq 3$, and so we can assume that $d(m, n)=3$. But then, again by Corollary 4.2 , we know that $m$ and $n$ lie in $\operatorname{cd}(G \mid M)$ and that their distance in $\mathcal{G}(G \mid M)$ is 3. Since $M$ is solvable and $M<N$, the inductive hypothesis guarantees that if $\mathcal{G}(G \mid M)$ is not connected, then no two vertices in the same component can have distance exceeding 2 . We conclude, therefore, that $\mathcal{G}(G \mid M)$ is connected. By hypothesis, however, $\mathcal{G}(G \mid N)$ is not connected, and thus we can choose $k \in \operatorname{cd}(G \mid N)$ in a different component from $m$. But then $k \notin \operatorname{cd}(G \mid M)$, and we conclude that $k \in \operatorname{cd}((G / M) \mid(N / M))$. Since $m$ is a $p^{\prime}$-number, it follows from Theorem 4.1 that $d(m, k) \leq 2$, and this is a contradiction.

\section{Structure When $N$ is a $p$-Group}

We now begin work toward proofs of Theorems B, C and D, which give structural information about $G$ and $N$ when $\mathcal{G}(G \mid N)$ is not connected. In this section, we consider the case where $N$ is an abelian $p$-group, and we prove Theorem $\mathrm{C}$. We also obtain a partial result in the case where $N$ is a nonabelian $p$-group.

We begin with a useful lemma, whose proof, unfortunately, requires material that is far from elementary. We will appeal to Lemma 3.5, which relies on the ItoMichler theorem and thus, ultimately, on the classification of simple groups. We also need the highly nontrivial Gluck-Wolf theorem, which asserts that if $N \triangleleft G$, $G / N$ is solvable and $\alpha \in \operatorname{Irr}(N)$, then $G / N$ has an abelian Hall $\pi^{\prime}$-subgroup, where $\pi$ is the set of all prime divisors of the numbers $\chi(1) / \alpha(1)$, where $\chi$ runs over $\operatorname{Irr}(G \mid \alpha)$. (See Theorem 12.9 of $[8$.) We will also use the far more elementary theorem of Ito that if $G=A B$, where $A$ and $B$ are abelian subgroups, then $G$ is metabelian. (See Satz VI.4.4 of [2].)

(5.1) Lemma. Let $\alpha, \beta \in \operatorname{Irr}(N)$, where $N \triangleleft G$, and suppose that every member of $\operatorname{cd}(G \mid \alpha)$ is coprime to every member of $\operatorname{cd}(G \mid \beta)$. If $G / N$ is solvable or if each of $\alpha$ and $\beta$ extends to its stabilizer in $G$, then $G / N$ is metabelian and all Sylow subgroups of $G / N$ are abelian.

Proof. Choose a set $\pi$ of prime numbers such that all members of $\operatorname{cd}(G \mid \alpha)$ are $\pi$ numbers and all members of $\operatorname{cd}(G \mid \beta)$ are $\pi^{\prime}$-numbers. We will show that $G / N$ has an abelian Hall $\pi$-subgroup and an abelian Hall $\pi^{\prime}$-subgroup. Then all Sylow subgroups 
of $G / N$ are abelian and since $G / N$ is the product of two abelian subgroups, it follows by Ito's theorem that $G / N$ is metabelian, as desired.

Assume first that $G / N$ is solvable. By the Clifford correspondence, the characters in $\operatorname{Irr}\left(G_{\alpha} \mid \alpha\right)$ induce to members of $\operatorname{Irr}(G \mid \alpha)$, and thus all of the degrees in $\operatorname{cd}\left(G_{\alpha} \mid \alpha\right)$ are $\pi$-numbers. It follows by the Gluck-Wolf theorem that $G_{\alpha} / N$ has an abelian Hall $\pi^{\prime}$-subgroup $A / N$. Also, since $\left|G: G_{\alpha}\right|$ divides each member of $\operatorname{cd}(G \mid \alpha)$, this index is a $\pi$-number, and thus $A / N$ is a Hall $\pi^{\prime}$-subgroup of $G / N$. Similarly, $G / N$ has an abelian Hall $\pi$-subgroup, and we are done in this case.

If $G / N$ is not solvable, then by hypothesis, we can assume that $\alpha$ and $\beta$ extend to their stabilizers in $G$. In this case, two applications of Lemma 3.5 guarantee the existence of the desired Hall subgroups.

(5.2) Corollary. Let $N \triangleleft G$ with $N \subseteq G^{\prime}$, and suppose that $\mathcal{G}(G \mid N)$ is not connected. If $N \subseteq S \triangleleft G$ and $S / N$ is solvable, then $S / N$ is metabelian.

Proof. Let $\alpha, \beta \in \operatorname{Irr}(N)$ lie under characters in $\operatorname{Irr}(G \mid N)$ with degrees lying in different components $X$ and $Y$ of $\mathcal{G}(G \mid N)$. By Corollary 2.3, all members of $\operatorname{cd}(G \mid \alpha)$ lie in $X$ and all members of $\operatorname{cd}(G \mid \beta)$ lie in $Y$. Now if $\theta \in \operatorname{Irr}(S \mid \alpha)$, then $\theta$ lies under some member of $\operatorname{Irr}(G \mid \alpha)$, and so $\theta(1)$ divides a member of $X$. Similarly, each member of $\operatorname{cd}(S \mid \beta)$ divides a member of $Y$, and thus all members of $\operatorname{cd}(S \mid \alpha)$ are coprime to all members of $\operatorname{cd}(S \mid \beta)$. We can now apply Lemma 5.1 in the group $S$ to conclude that $S / N$ is metabelian.

The following lemma is an easy consequence of Theorem A and Theorem 4.1.

(5.3) Lemma. Let $N$ be solvable, where $N \triangleleft G$ and $N \subseteq G^{\prime}$, and let $\mathcal{L}$ be the set of subgroups $L \subseteq N$ such that $N / L$ is a chief factor of $G$. Suppose that $\mathcal{G}(G \mid N)$ is not connected and let $Y$ be the set of degrees in $\operatorname{cd}(G \mid N)$ that are divisible by all prime divisors of $\left|N: N^{\prime}\right|$. Let $L \in \mathcal{L}$. If $\mathcal{G}(G \mid L)$ is not connected, then $Y \subseteq \operatorname{cd}(G \mid L)$ and if $\mathcal{G}(G \mid L)$ is connected, then $\operatorname{cd}(G \mid L) \subseteq Y$. Furthermore, there is at most one member $L \in \mathcal{L}$ such that $\mathcal{G}(G \mid L)$ is connected.

Proof. Let $X$ be the subset of $\operatorname{cd}(G \mid N)$ consisting of numbers coprime to $\left|N: N^{\prime}\right|$. By Theorem A, we know that $X$ and $Y$ are the connected components of $\mathcal{G}(G \mid N)$, and in particular, these sets are nonempty and they exhaust $\operatorname{cd}(G \mid N)$.

Given $L \in \mathcal{L}$, suppose that $\operatorname{cd}(G \mid L) \nsubseteq \subseteq$. We show in this case that $Y \subseteq \operatorname{cd}(G \mid L)$ and that $\mathcal{G}(G \mid L)$ is not connected. Let $p$ be the prime divisor of $|N: L|$ and choose $m \in \operatorname{cd}(G \mid L)$ with $m \notin Y$. Then $m \in X$, and so $p$ does not divide $m$. By Theorem 4.1, therefore, each member of $\operatorname{cd}((G / L) \mid(N / L))$ is at distance at most 2 from $m$ in $\mathcal{G}(G \mid N)$. Since $X$ is a connected component $\mathcal{G}(G \mid N)$ and $m \in X$, it follows that $\operatorname{cd}((G / L) \mid(N / L)) \subseteq X$, and hence $Y \subseteq \operatorname{cd}(G \mid L)$, as claimed. Also, $\mathcal{G}(G \mid L)$ is not connected in this case since $\operatorname{cd}(G \mid L)$ contains members of both $X$ and $Y$.

We see now that if $\mathcal{G}(G \mid L)$ is connected, then $\operatorname{cd}(G \mid L) \subseteq Y$. If, on the other hand, $\mathcal{G}(G \mid L)$ is not connected, then $\operatorname{cd}(G \mid L) \nsubseteq \subseteq Y$ since by the definition of the set $Y$, no two of its members are coprime. By the previous paragraph, therefore, we have $Y \subseteq \operatorname{cd}(G \mid L)$, as required.

Finally, we show that if $\mathcal{G}(G \mid L)$ is connected, then $L$ is the only member of $\mathcal{L}$ with this property. Choose $\chi \in \operatorname{Irr}(G \mid N)$ with $\chi(1) \in X$. Since $\operatorname{cd}(G \mid L) \subseteq Y$ in this case, we know that $\chi \notin \operatorname{Irr}(G \mid L)$, and thus $L \subseteq \operatorname{ker}(\chi) \cap N<N$. But $N / L$ 
is a chief factor of $G$, and thus $L=N \cap \operatorname{ker}(\chi)$. It follows that $L$ is unique, as required.

We can now prove Theorem $\mathrm{C}$, which we restate here.

(5.4) Theorem. Let $N$ be an abelian p-group, where $N \triangleleft G$ and $N \subseteq G^{\prime}$, and let $P \in \operatorname{Syl}_{p}(G)$. If $\mathcal{G}(G \mid N)$ is not connected, then all of the following occur:

(a) $P \triangleleft G$.

(b) $G / P$ and $P / N$ are abelian.

(c) Each member of $\operatorname{cd}(G \mid N)$ is either a power of $p$ or is coprime to $p$.

(d) $N$ is not central in $P$ but every minimal normal subgroup of $G$ contained in $G^{\prime}$ is central in $G$.

Proof. By Corollary 4.3, we know that the components of $\mathcal{G}(G \mid N)$ are exactly the set $X$ of $p^{\prime}$-degrees in $\operatorname{cd}(G \mid N)$ and the set $Y$ of degrees in $\operatorname{cd}(G \mid N)$ that are divisible by $p$. Working by induction on $|N|$, we first suppose that there is some chief factor $N / L$ of $G$ such that $\mathcal{G}(G \mid L)$ is not connected. Then conclusions (a), (b) and (d) are immediate by the inductive hypothesis applied with $L$ in place of $N$. Also, by Lemma 5.3, we know that $Y \subseteq \operatorname{cd}(G \mid L)$ in this case. Then $Y$ consists of powers of $p$ by the inductive hypothesis, and so conclusion (c) follows. We may suppose, therefore, that $\mathcal{G}(G \mid L)$ is connected whenever $N / L$ is a chief factor of $G$. It follows from Lemma 5.3 that $L$ is unique and that $\operatorname{cd}(G \mid L) \subseteq Y$.

By Corollary 2.3, we know that for each nonprincipal irreducible character $\lambda$ of $N$, either $\operatorname{cd}(G \mid \lambda) \subseteq X$ or $\operatorname{cd}(G \mid \lambda) \subseteq Y$, and since $X$ and $Y$ are nonempty, both possibilities actually occur. Choose nonprincipal characters $\mu, \nu \in \operatorname{Irr}(N)$ such that $\operatorname{cd}(G \mid \mu) \subseteq X$ and $\operatorname{cd}(G \mid \nu) \subseteq Y$. Since $\operatorname{cd}(G \mid L)$ contains no member of $X$, it follows that $L \subseteq \operatorname{ker}(\mu)$.

We argue that $\operatorname{core}_{G}\left(G_{\nu}\right) \subseteq G_{\mu}$. To see this, let $K \triangleleft G$ with $K \subseteq G_{\nu}$, and note that since $K$ stabilizes $\nu$, we have $[N, K] \subseteq \operatorname{ker}(\nu)<N$. But $[N, K] \triangleleft G$, and we conclude from the uniqueness of $L$ that $[N, K] \subseteq L \subseteq \operatorname{ker}(\mu)$, and thus $K$ stabilizes $\mu$, as desired.

Since $N$ is a $p$-group, the linear character $\mu \in \operatorname{Irr}(N)$ has $p$-power order. It lies under an irreducible character of $G$ having $p^{\prime}$-degree, and thus it follows by Corollary 2.2 that $\mu$ extends to $\hat{\mu} \in \operatorname{Irr}\left(G_{\mu} \mid \mu\right)$. In particular, $G_{\mu}<G$ since $\mu$ is linear and $N \subseteq G^{\prime}$, and thus $\mu$ does not extend to $G$. Also, we can apply Lemma 3.5 to obtain an abelian Hall $\pi^{\prime}$-subgroup $A / N$ of $G / N$ with $A \triangleleft G_{\mu}$, where $\pi$ is the set of all prime divisors of members of $X$.

Since $\left|G: G_{\nu}\right|$ divides some member of $Y$, this index is a $\pi^{\prime}$-number. It follows that $A G_{\nu}=G$, and we let $D=A \cap G_{\nu}$. Since $N \subseteq D \subseteq A$ and $A / N$ is abelian, we see that $D \triangleleft A$. It follows that $D^{G} \subseteq G_{\nu}$, and thus $D^{G} \subseteq \operatorname{core}_{G}\left(G_{\nu}\right) \subseteq G_{\mu}$.

Now $A / N$ is a normal Hall $\pi^{\prime}$-subgroup of $G_{\mu} / N$ and since $D^{G} \subseteq G_{\mu}$, we see that $D / N=A / N \cap D^{G} / N$ is a normal Hall $\pi^{\prime}$-subgroup of $D^{G} / N \triangleleft G / N$. It follows that $D / N \triangleleft G / N$, and hence $D \triangleleft G$. Also, since $A G_{\nu}=G$, we see that $\left|G_{\nu}: D\right|=|G: A|$ is a $\pi$-number. Finally, we let $Q \in \operatorname{Syl}_{p}(D)$. Then $N \subseteq Q$, and since $D / N$ is abelian, we have $Q \triangleleft D$, and thus $Q \triangleleft G$. Also, since $\left|G_{\nu}: D\right|$ is a $\pi$-number and $p \notin \pi$, we see that $Q \in \operatorname{Syl}_{p}\left(G_{\nu}\right)$.

Now $\mu$ extends to $\hat{\mu} \in \operatorname{Irr}\left(G_{\mu}\right)$ and we write $\alpha=(\hat{\mu})_{Q}$. Then $\alpha$ is invariant in $G_{\mu}$, and in fact $G_{\alpha}=G_{\mu}$ since $\mu=\alpha_{N}$. In particular, we see that $\hat{\mu}$ is an extension of $\alpha$ to its stabilizer in $G$. Next, we choose $\beta \in \operatorname{Irr}(Q \mid \nu)$ and we argue that $\beta$ also extends to its stabilizer in $G$. To see why this is so, observe that $Q \subseteq G_{\nu}$, and thus 
$\beta_{N}$ is a multiple of $\nu$ and we deduce that $G_{\beta} \subseteq G_{\nu}$. But $Q$ is a Sylow subgroup of $G_{\nu}$, and thus $Q$ is a Sylow subgroup of $G_{\beta}$, and it follows by a result of Gallagher that $\beta$ extends to $G_{\beta}$. (See Corollary 8.16 of [3].)

Now $\operatorname{cd}(G \mid \alpha) \subseteq X$ and $\operatorname{cd}(G \mid \beta) \subseteq Y$, and we know that every member of $X$ is coprime to every member of $Y$. Since we have seen that both $\alpha$ and $\beta$ extend to their stabilizers in $G$, it follows by Lemma 5.1 that $G / Q$ is metabelian, and in particular, $G$ is solvable. We can now apply Lemma 5.1 to the characters $\mu$ and $\nu$ since $\operatorname{cd}(G \mid \mu) \subseteq X$ and $\operatorname{cd}(G \mid \nu) \subseteq Y$, and we conclude that $G / N$ is metabelian, and so $G^{\prime} / N$ is abelian.

We next show that if $N \subseteq K \triangleleft G$, where $K / N$ is an $r$-group for some prime $r \neq p$, then a Sylow $r$-subgroup of $K$ is normal in $G$. To see this, let $R \in \operatorname{Syl}_{r}(K)$ and note that since $N$ is abelian, we have $N=[N, R] \times \mathbf{C}_{N}(R)$. Also, $[N, R]$ and $\mathbf{C}_{N}(R)$ are characteristic subgroups of $K$, and hence they are normal in $G$. But $\mathcal{G}(G \mid N)$ is not connected, and so it follows by Theorem 2.4 that one of the factors must be trivial. If $\mathbf{C}_{N}(R)=1$, then no nonprincipal linear character of $N$ is invariant in $K$, and thus all members of $\operatorname{cd}(G \mid N)$ are divisible by $r$. This is not the case, however, since $\mathcal{G}(G \mid N)$ is not connected, and so we conclude that $[N, R]=1$, and thus $R \triangleleft K$, and hence $R \triangleleft G$, as claimed.

If $G^{\prime}$ is not a $p$-group, we can choose a nontrivial Sylow $r$-subgroup $K / N$ of $G^{\prime} / N$ for some prime $r \neq p$. Then $K \triangleleft G$ since $G^{\prime} / N$ is abelian, and thus by the result of the previous paragraph, we see that $R \triangleleft G$, where $R \in \operatorname{Syl}_{r}(K)$. But $N \cap R=1$ and $R \cap G^{\prime}=R>1$, and this contradicts Theorem 2.6 since $\mathcal{G}(G \mid N)$ is not connected. It follows that $G^{\prime}$ is a $p$-group and thus $G^{\prime} \subseteq P$. Then $P \triangleleft G$ and $G / P$ is abelian, proving (a) and half of (b). Also, if $q$ is any prime divisor of a member of $Y$, then $q \in \pi^{\prime}$, and it follows that $A$ contains a full Sylow $q$-subgroup of $G$. In particular, by taking $q=p$, we see that $A \supseteq P$, and thus $P / N$ is abelian. This completes the proof of (b).

To prove (c), we suppose that some prime $r \neq p$ divides a member of $Y$. As we have seen, $A$ must contain a full Sylow $r$-subgroup $R$ of $G$, and we note that $R$ is abelian since $R \cap G^{\prime}=1$ because $G^{\prime}$ is a $p$-group. Also, $P \subseteq A$ and $A / N$ is abelian, and thus $[P, R] \subseteq N$ and $N R \triangleleft P R$. Now $N R / N$ is a normal Sylow $r$-subgroup of $P R / N$ and $P R \triangleleft G$ since $G / P$ is abelian. It follows that $N R \triangleleft G$, and thus by an earlier observation, we have $R \triangleleft G$. This is a contradiction, however, since $r$ divides some member of $Y \subseteq \operatorname{cd}(G)$, and thus by Ito's theorem, $G$ cannot have a normal abelian Sylow $r$-subgroup.

To prove (d), we first show that $N$ is not central in $P$. Otherwise, $P$ stabilizes the character $\nu$, and thus $P \subseteq G_{\nu}$ and we have $G_{\nu} \triangleleft G$ since $G / P$ is abelian. Then $G_{\nu}=\operatorname{core}_{G}\left(G_{\nu}\right) \subseteq G_{\mu}$, and since the indices $\left|G: G_{\mu}\right|$ and $\left|G: G_{\nu}\right|$ are coprime and $G_{\mu}<G$, this is a contradiction. It follows that $N$ is not central in $P$, as wanted.

Finally, suppose $V$ is minimal normal in $G$ and that $V \subseteq G^{\prime}$. Then $V \subseteq N$ by Corollary 2.7, and hence $[V, P]<V$ and we conclude that $[V, P]=1$ and $V \subseteq \mathbf{Z}(P)$. Since $N \nsubseteq \mathbf{Z}(P)$, it follows that $V<N$, and thus $V \subseteq L$. If $\tau \in \operatorname{Irr}(V)$ is nonprincipal, therefore, we have $\operatorname{cd}(G \mid \tau) \subseteq \operatorname{cd}(G \mid L) \subseteq Y$. Thus $\left|G: G_{\tau}\right|$ divides a member of $Y$, and so this index is a power of $p$. On the other hand, $P \subseteq G_{\tau}$ since $V$ is central in $P$, and it follows that $G_{\tau}=G$ and $[V, G] \subseteq \operatorname{ker}(\tau)<V$. Thus $[V, G]=1$, and the proof is complete.

(5.5) Corollary. Let $N$ be a minimal normal subgroup of $G$, where $N$ is solvable and $N \subseteq G^{\prime}$. Then $\mathcal{G}(G \mid N)$ is connected. 
Proof. Note that $N$ is an abelian $p$-group for some prime $p$, but $N$ does not satisfy conclusion (d) of Theorem 5.4. It follows that $\mathcal{G}(G \mid N)$ cannot fail to be connected.

We have been unable to obtain much useful information in the case where $N$ is a nonabelian $p$-group and $\mathcal{G}(G \mid N)$ is not connected. We do, however, have the following result, which we will use later, in the proof of Theorem D. To some extent, the proof of this theorem duplicates part of the proof of Theorem 5.4, but there are also some new ideas here.

(5.6) Theorem. Let $N$ be a nonabelian p-group, where $N \triangleleft G$ and $N \subseteq G^{\prime}$. Suppose that $\mathcal{G}(G \mid N)$ is not connected but that $\mathcal{G}(G \mid M)$ is connected whenever $M<N$ and $M \triangleleft G$. Then $N / \mathbf{Z}(N)$ is a chief factor of $G$.

Proof. As usual, we let $X$ and $Y$, respectively, be the subsets of $\operatorname{cd}(G \mid N)$ consisting of $p^{\prime}$-numbers and of multiples of $p$. Then $X$ and $Y$ are the connected components of $\mathcal{G}(G \mid N)$, and in particular, $X$ and $Y$ are nonempty. By hypothesis, $\mathcal{G}(G \mid L)$ is connected whenever $N / L$ is a chief factor of $G$, and hence by Lemma 5.3, there is a unique such chief factor $N / L$ and we have $\operatorname{cd}(G \mid L) \subseteq Y$. Also, since $\mathbf{Z}(N)<N$, we have $\mathbf{Z}(N) \subseteq L$ and we need to prove that $\mathbf{Z}(N)=L$. We assume that $\mathbf{Z}(N)<L$, and we work to obtain a contradiction.

Since $L$ is not central in $N$, we have $\mathbf{C}_{N}(L)<N$, and it follows that $\mathbf{C}_{N}(L) \subseteq L$. Let $H=\mathbf{C}_{G}(L) \triangleleft G$, and note that $[N, H] \subseteq N \cap H \subseteq L$, and thus $[N, H, H] \subseteq$ $[L, H]=1$. It follows from this that if $R \subseteq G$ is a $p^{\prime}$-subgroup that centralizes $L$, then $R$ centralizes $N$.

Now let $C$ be the intersection of the centralizers of all chief factors $U / V$ of $G$ such that $U \subseteq L$. If $R$ is a $p^{\prime}$-subgroup of $C$, then $R$ centralizes $L$, and thus $R$ centralizes $N$ by the result of the previous paragraph. It follows that every $p^{\prime}$-subgroup of $C$ acts trivially on $N / L$, and hence the group of automorphisms of $N / L$ induced by $C$ is a $p$-group. Therefore $L[N, C]<N$, and we conclude that $L[N, C]=L$, and thus $C$ acts trivially on $N / L$.

By Corollary 2.3, we can choose a nonprincipal character $\mu \in \operatorname{Irr}(N)$ such that $\operatorname{cd}(G \mid \mu) \subseteq X$. Then $L \subseteq \operatorname{ker}(\mu)$, and thus $C \subseteq G_{\mu}$ since $C$ centralizes $N / L$. Also, $\mu$ is linear and $\mu$ extends to $G_{\mu}$, and thus $G_{\mu} / N$ has a normal Sylow $p$-subgroup $P / N$, where $P \in \operatorname{Syl}_{p}(G)$. (We are using Lemma 3.5 here.) We also note that $\left|G: G_{\mu}\right|$ divides a member of $X$. Furthermore, since $N \subseteq G^{\prime}$ and $\mu$ is linear, we have $G_{\mu}<G$, and so $N / L \nsubseteq \mathbf{Z}(G / L)$.

Now let $U / V$ be a chief factor of $G$ with $U \subseteq L$. Then $P$ acts on the $p$-group $\operatorname{Irr}(U / V)$, and so we can choose a nonprincipal $P$-invariant linear character $\nu$ of $U / V$. Since $U \subseteq L$, we see that $\operatorname{cd}(G \mid \nu) \subseteq \operatorname{cd}(G \mid L) \subseteq Y$, and thus $\left|G: G_{\nu}\right|$ divides a member of $Y$. But $\left|G: G_{\mu}\right|$ divides a member of $X$, and thus these indices are coprime and $G=G_{\mu} G_{\nu}$.

We have $P \triangleleft G_{\mu}$ and $P \subseteq G_{\nu}$, and it follows that $P^{G} \subseteq G_{\nu}$, and hence $\left[U, P^{G}\right] \subseteq$ $\operatorname{ker}(\nu)$. Also, of course, $V \subseteq \operatorname{ker}(\nu)$, and thus $V \subseteq V\left[U, P^{G}\right] \subseteq \operatorname{ker}(\nu)<U$. Since $V\left[U, P^{G}\right] \triangleleft G$ and $U / V$ is a chief factor of $G$, we see that $\left[U, P^{G}\right] \subseteq V$, and thus $P^{G}$ centralizes the chief factor $U / V$.

We have now shown that $P^{G}$ centralizes each chief factor $U / V$ of $G$ with $U \subseteq L$, and thus $P^{G} \subseteq C \subseteq G_{\mu}$. But $P^{G}$ contains all Sylow $p$-subgroups of $G$ and $P$ is the unique Sylow $p$-subgroup of $G_{\mu}$, and it follows that $P \triangleleft G$. 
We also claim that $L \subseteq P^{\prime}$. Otherwise, there exists a linear character $\lambda$ of $P$ such that $L \nsubseteq \operatorname{ker}(\lambda)$, and thus $\operatorname{cd}(G \mid \lambda) \subseteq Y$. But $\lambda^{G}$ has $p^{\prime}$-degree since $P \in \operatorname{Syl}_{p}(G)$, and thus some irreducible constituent of $\lambda^{G}$ must have $p^{\prime}$-degree, and that is a contradiction.

Next, we show that $G / N$ is metabelian. Choose nonprincipal irreducible characters $\alpha$ and $\beta$ of $N$ such that $\operatorname{cd}(G \mid \alpha) \subseteq X$ and $\operatorname{cd}(G \mid \beta) \subseteq Y$. (Note that we are using Corollary 2.3 here.) Let $\hat{\alpha}, \hat{\beta} \in \operatorname{Irr}(P)$ lie over $\alpha$ and $\beta$, respectively, and note that $\operatorname{cd}(G \mid \hat{\alpha}) \subseteq X$ and $\operatorname{cd}(G \mid \hat{\beta}) \subseteq Y$. Because $P$ is a Sylow subgroup of $G$, it follows that $\hat{\alpha}$ and $\hat{\beta}$ extend to their stabilizers in $G$, and thus $G / P$ is metabelian by Lemma 5.1 applied to the characters $\hat{\alpha}$ and $\hat{\beta}$. But then $G$ is solvable, and we can apply Lemma 5.1 to the characters $\alpha$ and $\beta$. This time, we deduce that $G / N$ is metabelian, as claimed, and hence $G^{\prime} / N$ is abelian.

Suppose now that $P \subseteq K \triangleleft G$, where $K / P$ is an $r$-group for some prime $r$. We claim that $K$ centralizes $N / L$. To see this, let $U / V$ be an arbitrary chief factor of $G$ with $U \subseteq N$. Since $P$ acts trivially on $U / V$ and $K / P \triangleleft G / P$ is an $r$-group, we see that if $K$ does not centralize $U / V$, then the $G$-orbit of every nonprincipal linear character $\lambda$ of $U / V$ has size divisible by $r$. In particular, supposing that $K$ does not centralize the chief factor $N / L$, we see that $r$ divides the members of $\operatorname{cd}(G \mid \mu)$. In particular, $r$ divides some member of $X$, and thus $r$ divides no member of $Y$. If $U / V$ is an arbitrary chief factor of $G$ with $U \subseteq L$, then $\operatorname{cd}(G \mid U) \subseteq Y$ and it follows that $K$ acts trivially on $U / V$. Then $K \subseteq C$ and since $C \subseteq \mathbf{C}_{G}(N / L)$, we see that $K$ centralizes $N / L$. This is a contradiction, and so we have proved that $K$ really does centralize $N / L$.

We next show that $G / P$ is abelian. Otherwise, $G^{\prime} \nsubseteq P$ and we can choose $R \in \operatorname{Syl}_{r}\left(G^{\prime}\right)$ with $R>1$ and $r \neq p$. Then $R N / N \in \operatorname{Syl}_{r}\left(G^{\prime} / N\right)$ and since $G^{\prime} / N$ is abelian, we have $R N \triangleleft G$ and also $R P \triangleleft G$. Now $[P, R] \subseteq N$ and by the result of the previous paragraph, we have $[N, R] \subseteq L \subseteq P^{\prime}$. Thus $[P, R] \subseteq P^{\prime}$, and it follows that $R$ centralizes $P$, and thus $R \triangleleft R N$. We conclude that $R \triangleleft G$, and since $\mathcal{G}(G \mid N)$ is not connected, this contradicts Theorem 2.6 since $R \cap N=1$ and $1<R \subseteq G^{\prime}$.

We now know that $G / P$ is abelian. Since $N / L$ is not a central factor of $G$, we can choose a prime $r$ dividing $\left|G: \mathbf{C}_{G}(N / L)\right|$ and we let $K / P$ be a Sylow $r$-subgroup of $G / P$. Since $G / P$ is abelian, however, we have $K \triangleleft G$, and thus, as we have seen, $K$ centralizes $N / L$. This is our final contradiction.

\section{Structure When $N$ is Not a $p$-Group}

In this section, we prove Theorem D and we obtain Theorem B as a corollary. We begin with some lemmas, the first of which is known.

(6.1) Lemma. Let $B \subseteq A$, where $A$ is a finite abelian p-group, and assume that $\left\{x \in A \mid 1 \neq x^{p} \in B\right\} \subseteq B$. Then $B$ is a direct factor of $A$.

Proof. Since the result is trivial if $B=1$, we can assume that $B>1$, and we work by induction on $|B|$. We claim that $B \cap \Phi(A) \subseteq \Phi(B)$. To see this, let $b \in B \cap \Phi(A)$ and write $b=a^{p}$ with $a \in A$. If $b=1$, then certainly $b \in \Phi(B)$, and so we can assume that $1 \neq a^{p} \in B$, and we have $a \in B$, by hypothesis. Then $b=a^{p} \in \Phi(B)$, as claimed.

Since $B \cap \Phi(A) \subseteq \Phi(B)<B$, it follows that $B \nsubseteq \Phi(A)$. Then there exists a maximal subgroup $M$ of $A$ such that $B \nsubseteq M$, and thus $B M=A$. It is clear that the subgroup $B \cap M$ satisfies the hypothesis in $M$, and thus by the inductive 
hypothesis, we can write $M=(B \cap M) \times C$ for some subgroup $C \subseteq M$. Then $B \cap C=B \cap M \cap C=1$ and $B C=B(B \cap M) C=B M=A$. Thus $A=B \times C$, as wanted.

(6.2) Lemma. Let $S$ be a p-group that acts on a nontrivial $p^{\prime}$-group $M$. Let $K=\left\langle\mathbf{C}_{S}(m) \mid 1 \neq m \in M\right\rangle$ and assume that $K \subseteq \mathbf{Z}(S)$. Then $S$ has an abelian subgroup of index at most 2 and if $S$ is abelian, then $S / K$ is cyclic.

Proof. Work by induction on $|M|$. If $1<M_{0}<M$, where $M_{0}$ admits the action of $S$, write $K_{0}=\left\langle\mathbf{C}_{S}(m) \mid 1 \neq m \in M_{0}\right\rangle$ and note that $K_{0} \subseteq K \subseteq \mathbf{Z}(S)$. The result then follows by the inductive hypothesis since if $S / K_{0}$ is cyclic, then certainly $S / K$ is cyclic, too.

We can now assume that no nonidentity proper subgroup of $M$ admits the action of $S$. If $s \in S$ and $\mathbf{C}_{M}(s)>1$, then $s \in K \subseteq \mathbf{Z}(S)$, and thus $\mathbf{C}_{M}(s)$ admits $S$. We conclude that $\mathbf{C}_{M}(s)=M$ and $s$ acts trivially. It follows that the subgroup $K$ of $S$ is exactly the kernel of the action of $S$ and that the action of $S / K$ on $M$ is Frobenius. Since an abelian Frobenius complement is cyclic, it follows that if $S$ is abelian, then $S / K$ is cyclic, and there is nothing further to prove.

We can now assume that $S$ is nonabelian, and thus since $K \subseteq \mathbf{Z}(S)$, we see that $S / K$ is noncyclic. But $S / K$ is a $p$-group that is a Frobenius complement, and it follows that $p=2$ and that $S / K$ is generalized quaternion. But then there is a cyclic subgroup $C / K$ of $S / K$ with index 2. Also, since $K \subseteq \mathbf{Z}(C)$ and $C / K$ is cyclic, we see that $C$ is abelian. The proof is now complete.

(6.3) Corollary. Assume in the situation of Lemma 6.2 that $S$ is not abelian. Then $\mathbf{C}_{M}\left(S^{\prime}\right)=1$.

Proof. Let $N=\mathbf{C}_{M}\left(S^{\prime}\right)$ and suppose that $N>1$. Then $S / S^{\prime}$ acts on $N$ and $S^{\prime} \subseteq K$. Write $K_{0} / S^{\prime}=\left\langle\mathbf{C}_{S / S^{\prime}}(m) \mid 1 \neq m \in N\right\rangle$ and note that $K_{0} \subseteq K$. Since $S / S^{\prime}$ is abelian, we see by Lemma 6.2 that $S / K_{0} \cong\left(S / S^{\prime}\right) /\left(K_{0} / S^{\prime}\right)$ is cyclic. But $K_{0} \subseteq K \subseteq \mathbf{Z}(S)$, and thus $S$ is abelian, which is a contradiction.

Consider the following example. Let $G$ be the semidirect product of $G L(2,3)$ acting naturally on an elementary abelian group $M$ of order 9 . Let $N$ be the unique normal subgroup of $G$ of order 72 and note that $N \subseteq G^{\prime}$ and that $\operatorname{cd}(G \mid N)=$ $\{2,3,4,8,16\}$, so that $\mathcal{G}(G \mid N)$ is not connected. In fact, $N$ is minimal with the property that $N \triangleleft G$ and $\mathcal{G}(G \mid N)$ is not connected. To see this, note that there are just two nonidentity normal subgroups of $G$ that are properly contained in $N$. One of these is $M$, of order 9 , and the other is a subgroup $K$ of order 18 . It is easy to see that $\operatorname{cd}(G \mid M)=\{8,16\}$ and $\operatorname{cd}(G \mid K)=\{2,4,8,16\}$, and so the corresponding relative degree graphs are connected.

Now we abstract this example and define a pair $(N, G)$ to be exceptional if $N \triangleleft G$ and we have the following situation. We require that $N$ has an abelian normal 2complement $M$ and we write $Z / M=\mathbf{Z}(N / M)$. We require that $|N: Z|=4$, so that $N / Z$ is a Klein group. We let $C=\mathbf{C}_{G}(N / Z)$, so that $C$ is the kernel of the action of $G$ on $N / Z$. Since $N / Z$ is abelian, we see that $N \subseteq C$ and we require that $C / N$ has a normal Sylow 2-subgroup $P / N$. Then $M$ is a normal 2-complement in $P$ and $C / P$ has odd order, and thus $C$ is solvable by the odd-order theorem. Also, $G / C$ is embedded in the automorphism group of the Klein group $N / Z$, and thus $|G: C|$ divides 6 and we see that $G$ is solvable. 
We are now ready to state and prove a slightly strengthened version of Theorem D.

(6.4) Theorem. Let $N$ be solvable, where $N \triangleleft G$ and $N \subseteq G^{\prime}$. Let $p$ be a prime divisor of $\left|N: N^{\prime}\right|$ and assume that $N$ is not a p-group. If $N$ is minimal with the property that $\mathcal{G}(G \mid N)$ is not connected, then all of the following occur:

(a) $G$ is solvable.

(b) $N$ has a nilpotent normal $p$-complement $M$, and $M$ is abelian if $p=2$.

(c) $N / M$ is abelian except possibly when $p=2$ and $(N, G)$ is exceptional.

(d) $G / M$ has a normal Sylow p-subgroup except possibly when $p=2$ and $(N, G)$ is exceptional.

Proof. As usual, we let $X$ and $Y$, respectively, be the sets of $p^{\prime}$-numbers and of multiples of $p \operatorname{in} \operatorname{cd}(G \mid N)$. Since $\mathcal{G}(G \mid N)$ is not connected, we know by Corollary 4.3 that $X$ and $Y$ are exactly the connected components of $\mathcal{G}(G \mid N)$, and in particular, $X$ and $Y$ are nonempty. By hypothesis, we know that $\mathcal{G}(G \mid L)$ is connected whenever $N / L$ is a chief factor of $G$, and it follows from Lemma 5.3 that $L$ is unique, and thus $N / N^{\prime}$ is a $p$-group. Also, Lemma 5.3 guarantees that $\operatorname{cd}(G \mid L) \subseteq Y$. In other words, the members of $\operatorname{cd}(G \mid L)$ are all divisible by $p$, and it follows that $L$ has a normal $p$-complement $M$. (See Theorem D of 4 ] for this result of Y. Berkovich.) Then $M$ is a normal $p$-complement for $N$ and we have proved the existence part of (b).

Since $N / L$ is minimal normal in $G / L$, we know that $\mathcal{G}((G / L) \mid(N / L))$ is connected by Corollary 5.5. Since $X$ is nonempty and $\operatorname{cd}(G \mid L) \subseteq Y$, we see that $X \subseteq \operatorname{cd}((G / L) \mid(N / L))$, and hence equality must occur here.

If $\mu$ is a nonprincipal linear character of $N / L$, then $\operatorname{cd}(G \mid \mu) \subseteq X$, and thus $\left|G: G_{\mu}\right|$ divides a member of $X$. Also, as usual, $\mu$ extends to $G_{\mu}<G$ and $N / L \nsubseteq$ $\mathbf{Z}(G / L)$. Continuing to follow the familiar argument, we see by Lemma 3.5 that $G_{\mu} / N$ has a normal abelian Sylow $p$-subgroup $P / N$, and in fact, $P / N \in \operatorname{Syl}_{p}(G / N)$.

We next work to show that the stabilizer in $N$ of each nonprincipal character $\nu \in \operatorname{Irr}(M)$ is contained in $L$. To see this, write $U=N_{\nu}$ and $T=G_{\nu}$ and observe that $U=N \cap T \triangleleft T$. Note also that $|G: T|$ divides a member of $Y$ since $\operatorname{cd}(G \mid \nu) \subseteq Y$.

Since $U / M$ is a $p$-group and $M$ is a $p^{\prime}$-group, it follows that $\nu$ extends to $U$, and in fact, there is a unique extension $\hat{\nu}$ such that the determinant of $\hat{\nu}$ has $p^{\prime}$-order. (See Corollary 8.16 of [3].) Because of this uniqueness, we see that $\hat{\nu}$ is $T$-invariant. Now let $\mu \in \operatorname{Irr}(N / L)$ be nonprincipal and consider the character $\theta=\mu_{U} \hat{\nu} \in \operatorname{Irr}(U)$. We argue that $\theta$ is invariant in $T$ by showing that $\left|T: T_{\theta}\right|$ divides both a member of $X$ and a member of $Y$.

Let $\psi \in \operatorname{Irr}(T \mid \theta)$. Then $\psi \in \operatorname{Irr}(T \mid \nu)$ since $\theta_{M}=\nu$, and thus $\psi^{G} \in \operatorname{Irr}(G \mid \nu)$ by the Clifford correspondence. But $\operatorname{cd}(G \mid \nu) \subseteq Y$, and thus $\psi(1)$ divides a member of $Y$. Also, since $\psi \in \operatorname{Irr}(T \mid \theta)$, we see that $\left|T: T_{\theta}\right|$ divides $\psi(1)$, and so $\left|T: T_{\theta}\right|$ divides a member of $Y$.

Now write $D=G_{\mu} \cap T$. We previously saw that $\left|G: G_{\mu}\right|$ divides a member of $X$ and that $|G: T|$ divides a member of $Y$, and thus these indices are coprime. Then $T G_{\mu}=G$ and $|T: D|=\left|G: G_{\mu}\right|$ divides a member of $X$. But $D$ stabilizes both $\mu$ and $\hat{\nu}$, and thus $D$ stabilizes $\theta$ and it follows that $\left|T: T_{\theta}\right|$ divides $|T: D|$, and hence it divides a member of $X$. Combining this with the result of the previous paragraph, we conclude that $T_{\theta}=T$, and thus $\theta$ is invariant in $T$, as claimed.

By Gallagher's theorem, the characters $\theta=\mu_{U} \hat{\nu}$ and $\hat{\nu}$ uniquely determine $\mu_{U}$. Since both $\theta$ and $\hat{\nu}$ are invariant in $T$, it follows that $\mu_{U}$ is also invariant in $T$, 
and thus $[T, U] \subseteq \operatorname{ker}\left(\mu_{U}\right) \subseteq \operatorname{ker}(\mu)$. Also, $\left[G_{\mu}, U\right] \subseteq\left[G_{\mu}, N\right] \subseteq \operatorname{ker}(\mu)$. Since $G=$ $T G_{\mu}$ and $G_{\mu}$ normalizes $\operatorname{ker}(\mu)$, it follows that $[G, U]=\left[T G_{\mu}, U\right] \subseteq \operatorname{ker}(\mu)$. This containment holds for all choices of $\mu \in \operatorname{Irr}(N / L)$, and it follows that $[G, U] \subseteq L$, and thus $L U / L \subseteq \mathbf{Z}(G / L)$. But $N / L$ is a noncentral chief factor of $G$, and thus $(N / L) \cap \mathbf{Z}(G / L)$ is trivial. It follows that $L U=L$, and thus $U \subseteq L$, as wanted.

If $n \in N-L$, we have shown that $n$ fixes no nonprincipal irreducible character of $M$, and it follows that $n$ fixes no nonidentity conjugacy class of $M$. (We are using the fact that a group automorphism fixes equal numbers of irreducible characters and conjugacy classes. This is a consequence of a lemma of $\mathrm{R}$. Brauer that appears as Theorem 6.32 of [3].) We conclude, therefore, that $\mathbf{C}_{M}(n)=1$. Now let $S \in$ $\operatorname{Syl}_{p}(N)$, so that $S \cong N / M$ and $S$ acts on the $p^{\prime}$-group $M$. If we write $K=$ $\left\langle\mathbf{C}_{S}(m) \mid 1 \neq m \in M\right\rangle$, we have $K \subseteq L$ and $N / L$ is a homomorphic image of $S / K$. We also note that $M>1$ since by hypothesis, $N$ is not a $p$-group.

We next observe that if $N / M$ is abelian, then (c) certainly holds and $S$ is an abelian $p$-group. It follows by Lemma 6.2 that $S / K$ is cyclic, and thus $N / L$ is cyclic of order $p$. If we write $C=\mathbf{C}_{G}(N / L)$, we see that $N \subseteq C \triangleleft G$ and that $G / C$ is an abelian $p^{\prime}$-group. Also, $C \subseteq G_{\mu}$ for all $\mu \in \operatorname{Irr}(N / L)$, and thus $C / N$ has an abelian normal Sylow $p$-subgroup. It follows that $G / N$ has a normal Sylow $p$-subgroup, establishing $(\mathrm{d})$ in this case.

If $L=M$, then $K=1$ and hence the action of $S$ on $M$ is Frobenius, and so $M$ is nilpotent, and in fact, $M$ is abelian if $p=2$. In this case, therefore, conclusion (b) is established. Since $N / M$ is abelian in this situation, we know from the previous paragraph that (c) and (d) hold, and it remains is to prove (a): that $G$ is solvable. To accomplish this, note that $M>M^{\prime}$ since $M$ is a nontrivial nilpotent group, and so we can choose a nonprincipal linear character $\nu \in \operatorname{Irr}(M)$. Let $P / N$ be the normal Sylow $p$-subgroup of $G / N$ and choose $\alpha \in \operatorname{Irr}(P \mid \nu)$. Since $\nu$ is linear and $P / M$ is a $p$-group, it follows that $\alpha(1)$ is a power of $p$. Also, the linear character $\operatorname{det}(\alpha)$ has $p$-power order since $P / P^{\prime}$ is a $p$-group. (This is because $M \subseteq N^{\prime} \subseteq P^{\prime}$, where the first containment holds since $N$ is a Frobenius group with kernel $M$.) Since $p$ does not divide $\left|G_{\alpha}: P\right|$, it follows that $\alpha$ extends to $G_{\alpha}$. (See Corollary 8.16 of [3].) Also, if $\mu \in \operatorname{Irr}(N / M)$ is nonprincipal and $\beta \in \operatorname{Irr}(P \mid \mu)$, then since $P / M$ is a $p$-group and $p$ does not divide $\left|G_{\beta}: P\right|$, we see that $\beta$ extends to $G_{\beta}$. Since $\operatorname{cd}(G \mid \alpha) \subseteq \operatorname{cd}(G \mid \nu) \subseteq Y$ and $\operatorname{cd}(G \mid \beta) \subseteq \operatorname{cd}(G \mid \mu) \subseteq X$, it follows by Lemma 5.1 that $G / P$ is metabelian, and thus $G$ is solvable, as required.

We can now assume that $L>M$. We have

$$
\operatorname{cd}((G / M) \mid(N / M)) \supseteq \operatorname{cd}((G / M) \mid(L / M)),
$$

and this is a nonempty subset of $\operatorname{cd}(G \mid L) \subseteq Y$. Also, since $M \subseteq L$, it is clear that $\operatorname{cd}((G / M) \mid(N / M))$ contains

$$
\operatorname{cd}((G / L) \mid(N / L))=X .
$$

Since the set $\operatorname{cd}((G / M) \mid(N / M))$ contains members of $X$ and members of $Y$, it follows that the graph $\mathcal{G}((G / M) \mid(N / M))$ is not connected.

If $N / M$ is abelian, we have seen that (c) and (d) hold. By the result of the previous paragraph, we can apply Theorem 5.4 in the group $G / M$, and it follows that $G / M$ is solvable, and so if we can establish (b), it will follow that $G$ is solvable and (a) holds. We work now to prove (b). We must show, therefore, that $M$ is nilpotent and that it is abelian if $p=2$. Let $B=\operatorname{Irr}(N / L)$ and $A=\operatorname{Irr}(N / M)$, so that $B<A$ are abelian groups. Suppose that $\alpha \in A$ and that $1 \neq \alpha^{p}=\beta \in B$. 
Then $G_{\alpha} \subseteq G_{\beta}$ and since $\beta \in \operatorname{Irr}(N / L)$ is nonprincipal, we know that $\left|G: G_{\beta}\right|$ is a nontrivial divisor of a member of $X$. It follows that $\left|G: G_{\alpha}\right|$ cannot be a divisor of a member of $Y$, and thus $\operatorname{cd}(G \mid \alpha) \subseteq X$. We conclude from this that $L \subseteq \operatorname{ker}(\alpha)$, and thus $\alpha \in B$. It follows by Lemma 6.1 that $B$ is a direct factor of $A$.

Now it is easy to see that $L / M$ is a direct factor of $N / M$, and in particular, the group $S$ contains an element $s$ of order $p$ with $s \notin K$. Then $\mathbf{C}_{M}(s)=1$, and so $M$ is nilpotent and is abelian if $p=2$. This completes the proof of the theorem in the case where $N / M$ is abelian.

We can now assume that $N / M$ is nonabelian, and our task is to show that $p=2$ and the pair $(N, G)$ is exceptional. We must show, in particular, that $M$ is abelian. Also, if we write $Z / M=\mathbf{Z}(N / M)$ and $C=\mathbf{C}_{G}(N / Z)$, we must show that $|N: Z|=4$ and that $C / N$ has a normal Sylow 2-subgroup. Recall that this will imply that $G$ is solvable, and so conclusion (a) will follow.

Since $N / M$ is nonabelian, we know that $M<L$, and thus as we have seen, the graph $\mathcal{G}((G / M) \mid(N / M))$ is not connected. We wish to apply Theorem 5.6 to the group $G / M$, and so we need to check that $\mathcal{G}\left((G / M) \mid\left(N_{0} / M\right)\right)$ is connected whenever $N_{0} \triangleleft G$ and $N_{0}<N$. But $N_{0} \subseteq L$ in this case, and thus $\operatorname{cd}\left((G / M) \mid\left(N_{0} / M\right)\right) \subseteq \operatorname{cd}(G \mid L) \subseteq Y$, and so every one of these degrees is a multiple of $p$ and $\mathcal{G}\left((G / M) \mid\left(N_{0} / M\right)\right)$ is connected, as desired. It follows by Theorem 5.6 that $N / Z$ is a chief factor of $G$, and thus $Z=L$.

Then $K \subseteq \mathbf{Z}(S)$ and $S$ is nonabelian, and it follows by Lemma 6.2 that $S$ has an abelian subgroup with index 2. Thus $p=2$ and there exists an abelian subgroup $A / M$ of $N / M$ such that $|N: A|=2$. Also, since $N / M$ is nonabelian, we see that $A / M \supseteq \mathbf{Z}(N / M)=Z / M=L / M$. We know that $N / L$ is a noncentral chief factor of $G$, and thus $|N / L|>2$ and we have $L<A<N$. Therefore $A$ is not normal in $G$, and so the nonabelian group $N / M$ has at least two different abelian subgroups of index 2. It follows that the center $Z / M$ of $N / M$ has index 4 and that $(N / M)^{\prime}$ has order 2. Then $\left|S^{\prime}\right|=2$ and by Corollary 6.3 , we see that $\mathbf{C}_{M}\left(S^{\prime}\right)=1$. It follows that $M$ is abelian, as required. What remains in this case is to show that $C / N$ has a normal Sylow 2-subgroup. But this follows since $C \subseteq G_{\mu}$ for all linear characters $\mu$ of $N / L$. This completes the proof.

We can now prove Theorem B, which we restate here.

(6.5) Theorem. Let $N \triangleleft G$, where $N$ is solvable and $N \subseteq G^{\prime}$. If $\mathcal{G}(G \mid N)$ is not connected, then the Fitting height of $N$ is at most 3. Also, either $N^{\prime \prime}$ is nilpotent or there is an abelian normal subgroup of $G$ that has index 2 in $G^{\prime \prime \prime}$.

Proof. Note that in cases where we can show that $N^{\prime \prime}$ is nilpotent, the Fitting height of $N$ is clearly at most 3, and there will be nothing further to prove. Let $K \subseteq N$ with $K \triangleleft G$, where $K$ is minimal such that $\mathcal{G}(G \mid K)$ is not connected. Since $N$ is solvable, it follows from Corollary 5.2 that $N / K$ is metabelian, and thus $N^{\prime \prime} \subseteq K$. If $K$ is a $p$-group, we are done.

We now assume that $K$ is not a $p$-group, so that Theorem 6.4 applies. In particular, $G$ is solvable and $K$ has a nilpotent normal $p$-complement $M$. In this case, $G / K$ is metabelian by Corollary 5.2 , and we have $G^{\prime \prime} \subseteq K$. Now $N \subseteq G^{\prime}$, and thus $N^{\prime \prime} \subseteq G^{\prime \prime \prime}$, and so if $G^{\prime \prime \prime} \subseteq M$, we see that $N^{\prime \prime}$ is nilpotent, and we are done.

We can assume, therefore, that $G^{\prime \prime \prime} \nsubseteq M$. But $G^{\prime \prime} \subseteq K$, and so it follows that $K / M$ is nonabelian. We conclude from Theorem 6.4 that $p=2$ and that the pair 
$(K, G)$ is exceptional, and in particular, $M$ is abelian. Also, the center of the 2group $K / M$ has index 4 , and hence its derived subgroup $D / M$ has order 2 . Now $G^{\prime \prime} \subseteq K$, and hence $G^{\prime \prime \prime} \subseteq D$. We are assuming that $G^{\prime \prime \prime} \nsubseteq M$, and it follows that the abelian group $G^{\prime \prime \prime} \cap M$ has index 2 in $G^{\prime \prime \prime}$. Finally, we observe that $M$ and $K / M$ are nilpotent in this case and $N^{\prime} \subseteq G^{\prime \prime} \subseteq K$, and thus $N$ has Fitting height at most 3 .

\section{Diameters}

As usual, we assume that $N \triangleleft G$, where $N \subseteq G^{\prime}$, and $N$ is solvable. If $\mathcal{G}(G \mid N)$ is not connected, we know that all of the degrees in one component are divisible by $p$, where $p$ is a prime divisor of $\left|N: N^{\prime}\right|$, and so the diameter of that component is at most 1. Also, by Corollary 4.4, the diameter of the other component is at most 2. In this section we obtain upper bounds on the diameter of $\mathcal{G}(G \mid N)$ in the remaining case, where the graph is connected.

(7.1) Theorem. Let $N \triangleleft G$, where $N$ is solvable and $N \subseteq G^{\prime}$. Then no two vertices in $\mathcal{G}(G \mid N)$ can have finite distance exceeding 4 .

Proof. Assuming that the result is false, we can choose a counterexample where $N$ is minimal, and we can find $a, b \in \operatorname{cd}(G \mid N)$ with $d(a, b)=5$ in $\mathcal{G}(G \mid N)$. Let $N / M$ be a chief factor of $G$ and let $p$ be the prime divisor of $|N / M|$. Clearly, one of $a$ or $b$ is not divisible $p$, and so we can assume that $a$ is a $p^{\prime}$-number. Now choose $x, y \in \operatorname{cd}(G \mid N)$ such that $d(a, x)=3, d(x, y)=1$ and $d(y, b)=1$.

If $p$ does not divide $b$, then by Corollary 4.2 , we have $d(a, b) \leq 3$, which is not the case, and thus $p$ must divide $b$. But $x$ is coprime to $b$, and thus $x$ is a $p^{\prime}$-number. As $d(a, x)=3$ and $a$ and $x$ are $p^{\prime}$-numbers, it follows from Corollary 4.2 that both $a$ and $x$ lie in $\operatorname{cd}(G \mid M)$ and that their distance is at most 3 in $\mathcal{G}(G \mid M)$.

Since $a$ is a $p^{\prime}$-number in $\operatorname{cd}(G \mid M)$, it follows by Theorem 4.1 that the maximum possible distance in $\mathcal{G}(G \mid N)$ from $a$ to any member of $\operatorname{cd}((G / M) \mid(N / M))$ is at most 2. But $d(a, y)=4$ and $d(a, b)=5$, and we conclude that neither $y$ nor $b$ lies in $\operatorname{cd}((G / M) \mid(N / M))$, and so both $y$ and $b$ lie in $\operatorname{cd}(G \mid M)$. We see now that $a$ and $b$ lie in the same connected component of $\mathcal{G}(G \mid M)$ and that their distance in that subgraph is at most 5 . It follows that $d(a, b)=5$ in $\mathcal{G}(G \mid M)$, and this contradicts the minimality of $N$.

It seems likely that the correct maximum distance in Theorem 7.1 is 3 and not 4 , but we have been unable to prove this except in the case where $N$ is a $p$-group. We now begin work on this case.

We need the following technical lemma, which is related to results that have appeared in several papers on character degree graphs. (See, for example, the "Main Lemma" in [10, or Lemma 1 of [6], or Lemma 4 of [12].)

(7.2) Lemma. Let $N$ be a p-group and assume that $N^{\prime}$ is cyclic and central in $N$. Let $Y$ be a $p^{\prime}$-group that acts on $N$ and centralizes $N^{\prime}$ and assume that $Y$ has a normal abelian $r$-complement for some prime $r$ and that $Y=\mathbf{O}^{r^{\prime}}(Y)$. In addition, suppose that each linear character of $N$ is fixed by some Sylow $r$-subgroup of $Y$ and that if a linear character of $N$ is fixed by more than one Sylow r-subgroup of $Y$, then it is fixed by $Y$. Then $N=[N, Y] \times \mathbf{C}_{N}(Y)$.

Before we prove this, we recall that a partition of a group $G$ is a collection $\mathcal{P}$ of nonidentity subgroups such that $\bigcup \mathcal{P}=G$ and $U \cap V=1$ if $U, V \in \mathcal{P}$ are distinct. 
The partition is proper if it contains more than one subgroup. The following easy observation is well known.

(7.3) Lemma. Let $A$ be abelian and have a proper partition $\mathcal{P}$. Then $A$ is an elementary abelian p-group for some prime $p$.

Proof. Let $x \in A$ have prime order $p$ and let $P$ be the unique member of $\mathcal{P}$ that contains $x$. Let $y$ in $A$ be arbitrary and let $Q \in \mathcal{P}$ contain $x y$, so that $Q$ also contains $(x y)^{p}=y^{p}$. If $y^{p} \neq 1$, then $Q$ is the unique member of $\mathcal{P}$ that contains $y^{p}$, and thus $y \in Q$. Since $x y, y \in Q$, we see that $x \in Q$, and thus $Q=P$. We have thus shown that $y^{p}=1$ for all elements $y \in A$ except possibly for elements in $P$. Finally, choose $R \in \mathcal{P}$ different from $P$ and let $1 \neq z \in R$. We have shown that $z$ has order $p$, and now we replace $x$ by $z$ in the first argument to deduce that $y^{p}=1$ except possibly when $y \in R$. Since only the identity is in both $P$ and $R$, it follows that $y^{p}=1$ for all elements $y \in A$.

Proof of Lemma 7.2. Replacing $Y$ by $Y / \mathbf{C}_{Y}(N)$, we can assume that $Y$ acts faithfully on $N$. Write $A=N / N^{\prime}$ and note that since $|Y|$ is coprime to $|A|$, it follows that the actions of $Y$ on $A$ and on the set of linear characters of $N$ are permutationisomorphic, and thus each element of $A$ that is not fixed by $Y$ is fixed by exactly one Sylow $r$-subgroup of $Y$.

Since $|Y|$ is coprime to $|N|$, we have $N=[N, Y] \mathbf{C}_{N}(Y)$. Also, $[N, Y] \triangleleft N$ and we see that $\mathbf{C}_{N}(Y) \triangleleft N$ because $N^{\prime} \subseteq \mathbf{C}_{N}(Y)$. To prove that the product is direct, therefore, it suffices to show that $[N, Y] \cap \mathbf{C}_{N}(Y)=1$, or equivalently, that $\mathbf{C}_{U}(Y)=1$, where we have written $U=[N, Y]$. If we can show that $U$ is abelian, then since $[U, Y]=[N, Y, Y]=[N, Y]=U$ and $|U|$ and $|Y|$ are coprime, it will follow by Fitting's lemma that $\mathbf{C}_{U}(Y)=1$, as wanted. We assume, therefore, that $U$ is nonabelian, and we work to derive a contradiction.

Write $B=U / U^{\prime}$ and observe that $B$ is nontrivial. The kernel of the action of $Y$ on $B$ acts trivially on $N / U$, on $U / U^{\prime}$ and on $U^{\prime}$, and hence it acts trivially on $N$. Since the action of $Y$ on $N$ is faithful, however, it follows that $Y$ acts faithfully on $B$. Also, $[B, Y]=B$ and $B$ is abelian, and thus $\mathbf{C}_{B}(Y)=1$ and $\mathbf{C}_{U}(Y) \subseteq U^{\prime}$. Since $Y$ centralizes $N^{\prime}$, it follows that $N^{\prime} \cap U \subseteq \mathbf{C}_{U}(Y) \subseteq U^{\prime}$, and thus $U^{\prime}=N^{\prime} \cap U$. Thus $B$ is $Y$-isomorphic to a subgroup of $N / N^{\prime}=A$, and hence each element of $B$ is fixed by some Sylow $r$-subgroup of $Y$. Also, any element of $B$ that is fixed by more than one Sylow $r$-subgroup of $Y$ lies in $\mathbf{C}_{B}(Y)=1$, and so for each nonidentity element $b \in B$, there is a unique member $R \in \operatorname{Syl}_{r}(Y)$ such that $b \in \mathbf{C}_{B}(R)$. It follows that the subgroups $\mathbf{C}_{B}(R)$ are distinct as $R$ runs over $\operatorname{Syl}_{r}(Y)$ and these centralizers cover $B$ and their pairwise intersections are trivial. Furthermore, since the Sylow $r$-subgroups of $Y$ are conjugate, it follows that $Y$ acts transitively on the set $\mathcal{P}=\left\{\mathbf{C}_{B}(R) \mid R \in \operatorname{Syl}_{r}(Y)\right\}$, and hence the members of this set have equal orders.

If $Y$ is an $r$-group, then the set $\mathcal{P}$ contains just one member, namely $\mathbf{C}_{B}(Y)$. Since $\mathcal{P}$ covers $B>1$, it follows that $\mathbf{C}_{B}(Y)=B$. This is a contradiction since we know that $\mathbf{C}_{B}(Y)=1$ and $B>1$. Thus $Y$ is not an $r$-group, and since $Y=\mathbf{O}^{r^{\prime}}(Y)$, we see that $Y$ has more than one Sylow $r$-subgroup. In particular, $|\mathcal{P}|>1$, and hence $\mathcal{P}$ is a proper partition of $B$. By Lemma 7.3, we see that $B$ is an elementary abelian $p$-group and we can view $B$ as a $Y$-module over the field of order $p$. We also note that $r$ divides $|Y|$. 
We next argue that $B$ is simple as a $Y$-module. (We give an easy direct proof of this fact, although it can also be established by quoting the appropriate theorems.) Suppose that $B=B_{1} \dot{+} B_{2}$, where the $B_{i}$ are $Y$-invariant and nonzero. Let $s \in B_{1}$ and $t \in B_{2}$ be nonzero and let $R$ be the unique Sylow $r$-subgroup of $Y$ fixing $s+t$. Then $R$ fixes each of $s$ and $t$, and so we see that the unique Sylow $r$-subgroup of $Y$ that fixes $s$ also fixes every element $t \in B_{2}$. We deduce that $R$ acts trivially on $B_{2}$ and similarly, $R$ acts trivially on $B_{1}$. Thus $R$ centralizes $B$, and this is a contradiction since $R>1$ and the action of $Y$ on $B$ is faithful.

Now let $K$ be the abelian $r$-complement of $Y$ and decompose $B$ as a direct sum of its $K$-isotypic components. These summands are invariant under $K$ and are permuted transitively by $Y$; hence they are permuted transitively by each Sylow $r$-subgroup of $Y$. Choose a nonzero element $t$ in one of the isotypic components. Then $t$ is fixed by some Sylow $r$-subgroup $R$ of $Y$, and thus the isotypic component containing $t$ is also $R$-fixed. But $R$ acts transitively on the set of $K$-isotypic components, and it follows that there is just one such component, and thus $B$ is homogeneous as a $K$-module. Since the action of $K$ on $B$ is faithful and $K$ is abelian, it follows that this action is Frobenius and that $K$ is cyclic.

Write $k=|K|$ and $q=\left|\mathbf{C}_{B}(R)\right|$, where $R \in \operatorname{Syl}_{r}(Y)$, and observe that the partition $\mathcal{P}$ consists of $\left|\operatorname{Syl}_{r}(Y)\right|$ subgroups of order $q$. Since $Y=\mathbf{O}^{r^{\prime}}(Y)$, we have $[K, R]=K$, and thus $\mathbf{C}_{K}(R)=1$ and $\left|\operatorname{Syl}_{r}(Y)\right|=k$. We see, therefore, that $|B|-1=k(q-1)$.

Since $Y$ acts irreducibly on $B=U / U^{\prime}$ and $U^{\prime} \subseteq \mathbf{Z}(U)<U$, it follows that $\mathbf{Z}(U)=U^{\prime}$. Also, since $U / U^{\prime}$ is elementary abelian and $U^{\prime}$ is cyclic, it follows easily that $U$ is extraspecial, and hence $|B|=p^{2 e}$ for some integer $e>0$. The cyclic group $K$ of order $k$ has a Frobenius action on $U / U^{\prime}$ and $K$ centralizes $U^{\prime}$. It follows that either $k$ divides $p^{e}-1$ or $k$ divides $p^{e}+1$. (See Satz V.17.13 of [2].)

We have

$$
p^{e}+1 \geq k=\frac{|B|-1}{q-1}=\frac{p^{2 e}-1}{q-1},
$$

and thus $q-1 \geq p^{e}-1$. But $q$ is a power of $p$, and so we can write $q=p^{a}$, where $a \geq e$. Since $k>1$ is an integer, we see that $q-1=p^{a}-1$ is a proper divisor of $|B|-1=p^{2 e}-1$. It follows that $a$ divides $2 e$ and $a<2 e$. We know, however, that $a \geq e$, and we see, therefore, that $a=e$, and thus $q=p^{e}-1$ and $k=p^{e}+1$. But $k=\left|\operatorname{Syl}_{r}(Y)\right| \equiv 1 \bmod r$, and thus $r$ divides $k-1=p^{e}$, and we conclude that $r=p$. This is a contradiction, however, since we assumed that $|Y|$ and $p$ are coprime and we know that $r$ divides $|Y|$. This completes the proof.

We now present the main result of this section.

(7.4) Theorem. Let $N \triangleleft G$ be a p-group with $N \subseteq G^{\prime}$. Then no two vertices of $\mathcal{G}(G \mid N)$ can have finite distance exceeding 3.

Proof. Assume that the result is false and choose a counterexample where $|G|$ is minimal and where $N$ is minimal among normal subgroups of $G$ contained in $G^{\prime}$ and such that there exist $a, b \in \operatorname{cd}(G \mid N)$ having distance 4 in $\mathcal{G}(G \mid N)$. Also, since $p$ must fail to divide at least one of $a$ or $b$, we can assume that $a$ is a $p^{\prime}$-number.

If $a_{0} \in \operatorname{cd}(G \mid N)$ divides $a$, we see that $d\left(a_{0}, b\right) \geq 4$, and thus $d\left(a_{0}, b\right)=4$ by Theorem 7.1. We can thus replace $a$ by a divisor if necessary and assume that no proper divisor of $a$ lies in $\operatorname{cd}(G \mid N)$. Choose $x, y, z \in \operatorname{cd}(G \mid N)$ such that $d(a, x)=d(x, y)=d(y, z)=d(z, b)=1$. 
By Theorem 3.4, the greatest possible distance in $\mathcal{G}(G \mid N)$ between two $p^{\prime}$-degrees is 2 , and hence since $d(a, z)=3$ and $d(a, b)=4$, it follows that both $z$ and $b$ must be divisible by $p$. Also, $x$ and $y$ are coprime to $b$, and so they are $p^{\prime}$-numbers.

Now let $\alpha \in \operatorname{Irr}(N)$ lie under a character of degree $a$ in $\operatorname{Irr}(G \mid N)$. Since $\alpha(1)$ divides $a$, which is a $p^{\prime}$-number, we see that $\alpha$ has $p^{\prime}$-degree, and hence it is linear. It follows by Corollary 2.2 that $\alpha$ extends to $G_{\alpha}$, and thus by the Clifford correspondence $\left|G: G_{\alpha}\right|$ is a member of $\operatorname{cd}(G \mid N)$ that divides $a$. We thus have $\left|G: G_{\alpha}\right|=a$.

Every member of $\operatorname{cd}(G \mid \alpha)$ is divisible by $a$, and thus since $d(a, z)>2$, we see that all members of $\operatorname{cd}(G \mid \alpha)$ are coprime to $z$. Similarly, since $d(a, b)>2$, all members of $\operatorname{cd}(G \mid \alpha)$ are coprime to $b$. Since $\alpha$ extends to $G_{\alpha}$, it follows by Lemma 3.5 that for each prime divisor $r$ of $z$ or of $b$, a Sylow $r$-subgroup of $G / N$ is abelian and normal in $G_{\alpha} / N$. In particular, since $p$ divides $b$, we know that there exists $P \in \operatorname{Syl}_{p}(G)$ with $P \triangleleft G_{\alpha}$, and we see that $P / N$ is abelian.

Now let $\gamma \in \operatorname{Irr}(N)$ lie under a character of degree $y$ in $\operatorname{Irr}(G \mid N)$. Then $\gamma$ is linear since its degree divides the $p^{\prime}$-number $y$, and also $\gamma$ extends to $G_{\gamma}$ by Corollary 2.2. Write $c=\left|G: G_{\gamma}\right|$ and note that $c>1$ since $\gamma$ cannot extend to $G$. Since $c$ divides $y$, we see that $c$ is coprime to $p$ and to $a=\left|G: G_{\alpha}\right|$. Then $G=G_{\alpha} G_{\gamma}$, and thus $\left|G_{\alpha}: G_{\alpha} \cap G_{\gamma}\right|=\left|G: G_{\gamma}\right|=c$ is not divisible by $p$. But then $G_{\alpha} \cap G_{\gamma}$ contains the unique Sylow $p$-subgroup $P$ of $G_{\alpha}$, and so we have $P \subseteq G_{\gamma}$. Also, we note that if $r$ is any prime that divides no member of $\operatorname{cd}(G \mid \gamma)$, then reasoning as in the previous paragraph, we see that a Sylow $r$-subgroup of $G / N$ is normal in $G_{\gamma} / N$.

We work next to show that $P \triangleleft G$. Observe that if we can show that no member of $\operatorname{cd}(G \mid \gamma)$ is divisible by $p$, then $P \triangleleft G_{\gamma}$, and it will follow that $P \triangleleft G_{\alpha} G_{\gamma}=G$, as wanted. We assume now that $P$ is not normal in $G$, and we obtain a contradiction in this situation by deducing that the members of $\operatorname{cd}(G \mid \gamma)$ are not divisible by $p$.

Assuming that $P$ is not normal, we see that $P^{G}$ contains more than one Sylow $p$ subgroup of $G$, and thus $P^{G} \nsubseteq G_{\alpha}$. We conclude that $\left[N, P^{G}\right] \nsubseteq \operatorname{ker}(\alpha)$, and so if we write $K=\left[N, P^{G}\right]$ and $\lambda=\alpha_{K}$, it follows that $K \triangleleft G$ and $\lambda$ is a nonprincipal linear character of $K$. Since $\lambda$ lies under $\alpha$, we see that $a \in \operatorname{cd}(G \mid \lambda)$, and thus $\left|G: G_{\lambda}\right|$ divides $a$. Also, $\lambda$ extends to $G_{\lambda}$ by Corollary 2.2 , and thus $\left|G: G_{\lambda}\right| \in \operatorname{cd}(G \mid N)$ by the Clifford correspondence. But no proper divisor of $a$ lies in $\operatorname{cd}(G \mid N)$, and thus $a=\left|G: G_{\lambda}\right|$. Also $G_{\alpha} \subseteq G_{\lambda}$ since $\alpha_{K}=\lambda$, and since $\left|G: G_{\alpha}\right|=a=\left|G: G_{\lambda}\right|$, we conclude that $G_{\lambda}=G_{\alpha}$.

Recall that $G=G_{\alpha} G_{\gamma}$ and that $P \triangleleft G_{\alpha}$ and $P \subseteq G_{\gamma}$. It follows that $P^{G} \subseteq G_{\gamma}$, and thus $K=\left[N, P^{G}\right] \subseteq \operatorname{ker}(\gamma)$.

Now let $\tau=\alpha \gamma$. Since $K \subseteq \operatorname{ker}(\gamma)$, we have $\tau_{K}=\alpha_{K}=\lambda$, and thus $G_{\tau} \subseteq G_{\lambda}=$ $G_{\alpha}$. Since $G_{\tau}$ stabilizes $\tau=\alpha \gamma$ and we now know that it also stabilizes $\alpha$, it follows that $G_{\tau}$ stabilizes $\gamma$. Then $G_{\tau} \subseteq G_{\alpha} \cap G_{\gamma}$, and since the reverse containment is clear, we deduce that $G_{\tau}=G_{\alpha} \cap G_{\gamma}$. Then $\left|G: G_{\tau}\right|=\left|G: G_{\alpha}\right|\left|G: G_{\gamma}\right|=a c$, where the first equality follows since we know that $G=G_{\alpha} G_{\gamma}$. Also, since $\alpha$ extends to $G_{\alpha}$ and $\gamma$ extends to $G_{\gamma}$, we see that $\tau=\alpha \gamma$ extends to $G_{\alpha} \cap G_{\gamma}=G_{\tau}$. It follows by the Clifford correspondence that $a c \in \operatorname{cd}(G \mid N)$.

Suppose now that some member $m \in \operatorname{cd}(G \mid \gamma)$ is divisible by $p$. Then in $\mathcal{G}(G \mid N)$, we have $d(m, b) \leq 1$. Also, $m$ is divisible by $c=\left|G: G_{\gamma}\right|$, and thus $d(a c, m) \leq 1$. Clearly, $d(a, a c) \leq 1$, and we deduce that $d(a, b) \leq 3$, which is a contradiction. We conclude that no member of $\operatorname{cd}(G \mid \gamma)$ is divisible by $p$, and as we have seen, this completes the proof that $P \triangleleft G$. 
The $p^{\prime}$-group $G / P$ acts on the abelian $p$-group $\operatorname{Irr}(N /[N, P])$, and so it follows by Lemma 3.1 that if $\mu$ and $\nu$ are $P$-invariant linear characters of $N$ lying in $G$ orbits of coprime sizes $m$ and $n$, respectively, then the orbit of $\mu \nu$ has size $m n$. In particular, since $\alpha$ and $\gamma$ are $P$-invariant and lie in orbits of sizes $a$ and $c$, we see that if we set $\tau=\alpha \gamma$, then $\left|G: G_{\tau}\right|=a c$, and hence $G_{\tau}=G_{\alpha} \cap G_{\gamma}$. (We have seen this before, but previously it was established using the "incorrect" assumption that $P$ was not normal in $G$.) Also, $\alpha$ and $\gamma$ extend to $G_{\alpha}$ and $G_{\gamma}$, respectively, and it follows that $\tau$ extends to $G_{\tau}$. By the Clifford correspondence, therefore, we have $a c=\left|G: G_{\tau}\right| \in \operatorname{cd}(G \mid N)$. We have $d(a c, y) \leq 1$ since $c$ divides $y$ and also $d(a, a c) \leq 1$, and thus we can assume that $x=a c$.

Now fix some prime divisor $r$ of $z$ or $b$ with $r \neq p$. Recall that we saw that some Sylow $r$-subgroup of $G / N$ is normal in $G_{\alpha} / N$, and thus we can choose $R \in \operatorname{Syl}_{r}(G)$ such that $N R \triangleleft G_{\alpha}$. Also, since $P \triangleleft G_{\alpha}$ and $P \cap N R=N$, we see that $R$ centralizes $P / N$. But $P \triangleleft G$ and it follows that every Sylow $r$-subgroup of $G$ centralizes $P / N$.

Recall that $P / N$ is abelian, and so we have $P^{\prime} \subseteq N$. We claim that each linear character of $N / P^{\prime}$ is fixed by some Sylow $r$-subgroup of $G$. To see this, let $\lambda$ be a nonprincipal linear character of $N / P^{\prime}$ and observe that $\lambda$ is $P$-invariant. In fact, $\lambda$ extends to $P$, and thus $\operatorname{cd}(G \mid \lambda)$ contains a $p^{\prime}$-number. Then $\lambda$ extends to $G_{\lambda}$ by Corollary 2.2, and thus $\left|G: G_{\lambda}\right| \in \operatorname{cd}(G \mid N)$, and we write $m=\left|G: G_{\lambda}\right|$. Our goal is to show that $r$ does not divide $m$.

By assumption, $r$ divides $z$ or $b$, and so if $r$ divides $m$, we have $d(m, b) \leq 2$ in $\mathcal{G}(G \mid N)$. It follows that $d(a, m) \geq 2$, and thus $a$ and $m$ are coprime. The linear characters $\alpha$ and $\lambda$ are $P$-invariant and lie in $G / P$-orbits having coprime sizes, and it follows that $\alpha \lambda$ lies in an orbit of size $a m$. We conclude that some multiple $n$ of $a m$ lies in $\operatorname{cd}(G \mid N)$ and we see that $r$ divides $n$. But then $d(a, n) \leq 1$ and $d(n, b) \leq 2$, and this is a contradiction. We conclude that $r$ does not divide $m$, as required.

We argue next that $G / N$ does not have a normal Sylow $r$-subgroup. Otherwise, $N R \triangleleft G$, where $R \in \operatorname{Syl}_{r}(G)$, and we get the same group $N R$ for each choice of the subgroup $R \in \operatorname{Syl}_{r}(G)$. We showed that each linear character of $N / P^{\prime}$ is fixed by some Sylow $r$-subgroup of $G$, and thus is fixed by $N R$ and hence by $R$. It follows that $R$ centralizes $N / P^{\prime}$ and since $r \neq p$ and we saw previously that $R$ centralizes $P / N$, it follows that $R$ centralizes $P / P^{\prime}$, and thus $R$ centralizes $P$. We conclude that $R \triangleleft N R$, and thus $R \triangleleft G$. But $r$ divides some member of $\operatorname{cd}(G)$, and it follows by Ito's theorem that the normal Sylow $r$-subgroup $R$ of $G$ cannot be abelian. Thus $R \cap G^{\prime}>1$ and this contradicts Theorem 2.6 since the diameter of $\mathcal{G}(G \mid N)$ exceeds 3 . We have now shown that as claimed, $G / N$ does not have a normal Sylow $r$-subgroup.

It now follows that $G / P$ does not have a normal Sylow $r$-subgroup since otherwise $P R \triangleleft G$, where $R \in \operatorname{Syl}_{r}(G)$. But $R$ centralizes $P / N$, and it follows that $N R \triangleleft P R$ and thus $N R \triangleleft G$, which we know is not the case.

Next, we argue that $b$ is a power of $p$. Otherwise, we can choose the prime $r$ so that it divides $b$. (Recall that the only conditions on $r$ were that it divides $b$ or $z$ and that it is different from $p$.) We have seen that some Sylow $r$-subgroup of $G / N$ is normal in $G_{\alpha} / N$ and we will also show that some Sylow $r$-subgroup of $G / N$ is normal in $G_{\gamma} / N$. We know, however, that $G_{\alpha} G_{\gamma}=G$, and it follows easily from this that $G / N$ has a normal Sylow $r$-subgroup. This is false, however, and this contradiction will show that $b$ is a power of $p$, as desired. 
Since $\gamma$ extends to $G_{\gamma}$, we see that to show that a Sylow $r$-subgroup of $G / N$ is normal in $G_{\gamma} / N$, it suffices to show that $\operatorname{cd}(G \mid \gamma)$ contains no multiple of $r$. We suppose, therefore, that there exists $n \in \operatorname{cd}(G \mid \gamma)$ divisible by $r$. Then $d(n, b) \leq 1$ in $\mathcal{G}(G \mid N)$, and also $d(x, n) \leq 1$ since $c$ divides both $n$ and $x$. This is a contradiction since $d(x, b)=3$, and this completes the proof that $b$ is a power of $p$. In particular, we know that the prime $r$ divides $z$, and in fact, since $p$ does not divide $y$, we can assume from now on that $r$ is a common prime divisor of $y$ and $z$.

Next, we show that $G$ is solvable and that, in fact, $G / N$ is metabelian. Let $\mu \in \operatorname{Irr}(P)$ lie under a character in $\operatorname{Irr}(G \mid N)$ of degree $a$ and let $\nu \in \operatorname{Irr}(P)$ lie under a character in $\operatorname{Irr}(G \mid N)$ of degree $b$. If $\chi \in \operatorname{Irr}(G \mid \mu)$ and $\psi \in \operatorname{Irr}(G \mid \nu)$, then $\chi(1)$ and $\psi(1)$ lie in $\operatorname{cd}(G \mid N)$ and by Corollary 2.3, they are joined to $a$ and $b$, respectively, in $\mathcal{G}(G \mid N)$. Since $d(a, b)=4$, it follows that $\chi(1)$ and $\psi(1)$ are coprime. Also, because $P$ is a normal Sylow subgroup of $G$, we see that $\mu$ and $\nu$ extend to their stabilizers in $G$. By Lemma 5.1, therefore, $G / P$ is metabelian, and hence $G$ is solvable. We can thus apply Lemma 5.1 again, where this time, $\mu$ and $\nu$ are chosen to lie in $\operatorname{Irr}(N)$ rather than in $\operatorname{Irr}(P)$. (This time, we do not know that $\mu$ and $\nu$ extend to their stabilizers in $G$, but since $G$ is solvable, this does not matter.) We conclude that $G / N$ is metabelian, as claimed, and thus $G^{\prime} / N$ is abelian.

Now suppose that $q$ is a prime divisor of $\left|G^{\prime} / N\right|$ different from $p$ and let $Q / N \in$ $\operatorname{Syl}_{q}\left(G^{\prime} / N\right)$. Then $Q \subseteq G^{\prime}$ and since $G^{\prime} / N$ is abelian, we see that $Q \triangleleft G$. Now $d(a, b)>3$ in $\mathcal{G}(G \mid N)$, and so by Theorem 4.1, we see that one of $a$ or $b$ must be divisible by $q$. Since $b$ is a power of $p$, it follows that $q$ divides $a$, and this shows that every prime divisor of $\left|G^{\prime}\right|$ other than $p$ must divide $a$. In particular, we see that any subgroup of $G$ with index coprime to ap must contain $G^{\prime}$.

Since $b$ is a power of $p$, a character in $\operatorname{Irr}(G \mid N)$ of degree $b$ must restrict irreducibly to $P$, and it follows by Gallagher's theorem that if $f \in \operatorname{cd}(G / P)$, then $b f \in \operatorname{cd}(G \mid N)$ and $d(b f, b) \leq 1$. Since $d(x, b)=3$, we see that $d(x, b f) \geq 2$, and so $f$ and $x$ are coprime. By the Ito-Michler theorem, it follows that $G / P$ has an abelian normal Hall $\pi$-subgroup $U / P$, where $\pi$ is the set of prime divisors of $x$. (Actually, since $G$ is solvable, we are really using Ito's original theorem here, and not Michler's extension of it to nonsolvable groups.) Then $U$ is a normal Hall $(\pi \cup\{p\})$-subgroup of $G$ and $|G: U|$ is coprime to $p x$. Also, since $a$ divides $x$, it follows from the result of the previous paragraph that $G^{\prime} \subseteq U$. We also mention that $r$ does not divide $|U|$ since $r \neq p$ and $r$ does not divide $x$. (The reason for the latter assertion is that $r$ divides $z$ and $d(x, z)=2$ in $\mathcal{G}(G \mid N)$.)

As before, let $R \in \operatorname{Syl}_{r}(G)$. Let $H=U R$ and note that $H \triangleleft G$ since $G^{\prime} \subseteq U$. Let $M=N \cap H^{\prime} \triangleleft G$ and observe that $P^{\prime} \subseteq M$ since $P^{\prime} \subseteq N$ and $P \subseteq U \subseteq H$. Then $G / M$ has a normal abelian Sylow $p$-subgroup, and in particular, $N / M$ is abelian. Also, no irreducible character of $G / M$ has degree divisible by $p$, and so $z$ and $b$ lie in $\operatorname{cd}(G \mid M)$.

We claim that $a, x$ and $y$ also lie in $\operatorname{cd}(G \mid M)$. To see why this is so, let $\chi \in$ $\operatorname{Irr}(G \mid N)$ have one of these degrees and suppose that $M \subseteq \operatorname{ker}(\chi)$. Let $\lambda$ be an irreducible constituent of $\chi_{N}$, so that $\lambda$ is nonprincipal. Also, $\lambda$ is linear since $\chi(1)$ is a $p^{\prime}$-number, and it follows that $\lambda$ extends to $G_{\lambda}$, and thus $\left|G: G_{\lambda}\right|>1$. Since $N \subseteq H$, we have $[N, H] \subseteq N \cap H^{\prime}=M \subseteq \operatorname{ker}(\lambda)$, and thus $H \subseteq G_{\lambda}$. Then $U \subseteq G_{\lambda}$, and hence no prime divisor of $x$ can divide $\left|G: G_{\lambda}\right|$, and in particular, no prime divisor of $a$ can divide $\left|G: G_{\lambda}\right|$ since $a$ divides $x$. But $\left|G: G_{\lambda}\right|$ is a nontrivial 
divisor of $\chi(1)$, and so we cannot have $\chi(1)=x$ or $\chi(1)=a$, and this shows that $x, a \in \operatorname{cd}(G \mid M)$. Also to see that $y \in \operatorname{cd}(G \mid M)$, it suffices to show that we cannot have $\lambda=\gamma$. This follows since $\left|G: G_{\gamma}\right|=c$ and $c$ divides $x$.

We have now seen that $a, x, y, z, b \in \operatorname{cd}(G \mid M)$, and thus $a$ and $b$ lie in the same connected component of $\mathcal{G}(G \mid M)$ and their distance in this subgraph of $\mathcal{G}(G \mid N)$ cannot be less than their distance in $\mathcal{G}(G \mid N)$, which is 4 . It follows by the minimality of $N$ that $M=N$, and thus $N \subseteq H^{\prime}$.

Now $|G: H|$ is coprime to $a, x$ and $b$, and since $H \triangleleft G$, it follows that irreducible characters of $G$ with these degrees restrict irreducibly to $H$, and hence $a, x, b \in$ $\operatorname{cd}(H \mid N)$. Also, we see that $\operatorname{cd}(H \mid N)$ contains a divisor $y_{1}$ of $y$ and a divisor $z_{1}$ of $z$ such that $y / y_{1}$ and $z / z_{1}$ divide $|G: H|$. Recall that $c$ divides $y$ and since $c$ divides $x$, it is coprime to $|G: H|$, and thus $c$ divides $y_{1}$ and it follows that $x$ and $y_{1}$ are joined in $\mathcal{G}(H \mid N)$. Also, $r$ divides both $y$ and $z$ and does not divide $|G: H|$, and thus $r$ divides both $y_{1}$ and $z_{1}$, which, therefore are also joined in $\mathcal{G}(H \mid N)$. Finally, since $p$ divides $z$ but does not divide $|G: H|$, we see that $p$ divides $z_{1}$, and thus $z_{1}$ and $b$ are joined in $\mathcal{G}(H \mid N)$. It follows that $a$ and $b$ are in the same connected component of $\mathcal{G}(H \mid N)$. Furthermore, their distance in this graph cannot be less than 4 since every member of $\operatorname{cd}(H \mid N)$ divides some member of $\operatorname{cd}(G \mid N)$. It follows that $d(a, b)=4$ in $\mathcal{G}(H \mid N)$ and since $N \subseteq H^{\prime}$, the minimality of the counterexample $G$ shows that $H=G$. We conclude that $G / P$ has the normal abelian $r$-complement $U / P$.

If $\lambda$ is a linear character of $N$ that does not extend to $P$, then every member of $\operatorname{cd}(G \mid \lambda)$ is divisible by $p$. These numbers are thus at a distance at most 1 from $b$ in $\mathcal{G}(G \mid N)$, and hence they are coprime to $a$. Now let $V / N$ be the $p$-complement of the abelian group $G^{\prime} / N$ and recall that we know that every prime divisor of $|V / N|$ divides $a$. Since $V \triangleleft G$ and $\left|G: G_{\lambda}\right|$ is coprime to $a$, it follows that $V \subseteq G_{\lambda}$ and $V$ stabilizes $\lambda$.

Now $\alpha$ extends to $G_{\alpha}$, and so $\alpha$ extends to $P$, and since $\lambda$ does not extend to $P$, it follows that $\alpha \lambda$ also does not extend to $P$. Reasoning as we did with $\lambda$, we see that $V$ also stabilizes $\alpha \lambda$, and it follows that $V$ stabilizes $\alpha$. Then $G_{\alpha} \supseteq P V \supseteq G^{\prime}$, and it follows that $G_{\alpha} \triangleleft G$. This is not the case, however, because we know that a Sylow $r$-subgroup of $G / N$ is normal in $G_{\alpha} / N$, but it is not normal in $G / N$. This contradiction shows that every linear character of $N$ extends to $P$, and thus $N^{\prime}=P^{\prime}$. It follows also that each such character lies under an irreducible character of $G$ having $p^{\prime}$-degree, and hence by Corollary 2.2, every linear character of $N$ extends to its stabilizer in $G$.

Recall that we saw earlier that each linear character of $N / P^{\prime}$ is fixed by some Sylow $r$-subgroup of $G$. It now follows that every linear character of $N$ is fixed by such a Sylow subgroup. Also, since $P$ acts trivially on the set of linear characters of $N$, we see that we have an action of $\bar{G}=G / P$ on these linear characters and it follows that each linear character of $N$ is fixed by some Sylow $r$-subgroup of $\bar{G}$.

We argue next that if $\lambda$ is a linear character of $N$ that is stabilized by more than one Sylow $r$-subgroup of $\bar{G}$, then $\lambda$ is stabilized by all Sylow $r$-subgroups of $\bar{G}$. The stabilizer of $\lambda$ in $\bar{G}$ is $G_{\lambda} / P$, and by assumption, this group fails to have a normal Sylow $r$-subgroup. Then a Sylow $r$-subgroup of $G_{\lambda} / N$ is not normal, and hence since $\lambda$ extends to $G_{\lambda}$, it follows by Lemma 3.5 that $\mathrm{cd}(G \mid \lambda)$ contains a multiple $m$ of $r$. Then $d(m, z) \leq 1$ in $\mathcal{G}(G \mid N)$, and hence $d(a, m) \geq 2$ and we see that $a$ and $m$ are coprime. But $\left|G: G_{\lambda}\right|$ divides $m$, and so this index is coprime to $a$. We know 
that $\left|G: G_{\lambda}\right|$ is also coprime to $p$, and as we have seen, this implies that $G^{\prime} \subseteq G_{\lambda}$, and thus $G_{\lambda} \triangleleft G$. But $G_{\lambda}$ contains a Sylow $r$-subgroup of $G$, and it follows that it contains all Sylow $r$-subgroups of $G$. We see from this that every Sylow $r$-subgroup of $\bar{G}$ stabilizes $\lambda$, as claimed.

Now let $K / L$ be a chief factor of $G$ with $K \subseteq P^{\prime}$ and let $\tau$ be an arbitrary nonprincipal linear character of $K / L$. Then $\tau$ does not extend to $P$, and hence every degree in $\operatorname{cd}(G \mid \tau)$ is divisible by $p$. Also, $\operatorname{cd}(G \mid \tau) \subseteq \operatorname{cd}(G \mid N)$ since $K \subseteq P^{\prime} \subseteq N$, and thus since $d(x, b)=3$ and $b$ is divisible by $p$, it follows that all degrees in $\operatorname{cd}(G \mid \tau)$ are coprime to $x$ and we see that $\left|G: G_{\tau}\right|$ is coprime to $x$. Also, $P$ centralizes the chief factor $K / L$ and thus $P \subseteq G_{\tau}$, and it follows that $U \subseteq G_{\tau}$, where we recall that $U / P$ is the normal Hall $\pi$-subgroup of $G / P$, where $\pi$ is the set of prime divisors of $x$. If $Q$ is a Hall $\pi$-subgroup of $G$, therefore, we see that $Q$ fixes every linear character of $K / L$, and thus $Q$ centralizes $K / L$.

Let $C=\mathbf{C}_{G}\left(P^{\prime}\right)$ and note that $Q \subseteq C$ since $Q$ centralizes all $G$-chief factors in $P^{\prime}$ and $p$ does not divide $|Q|$. We claim that $N \subseteq C$. Otherwise, since $C \triangleleft G$, we have $[N, C] \subseteq N \cap C<N$ and we can choose a nonprincipal $C$-invariant linear character $\mu$ of $N$. Then $\mu$ is stabilized by $P$, by $Q$ and by some Sylow $r$-subgroup of $G$, and thus $\mu$ is $G$-invariant since $P Q R=G$. But $\mu$ extends to $G_{\mu}=G$, and this is a contradiction since $N \subseteq G^{\prime}$. This shows that $N \subseteq C$, as claimed, and thus $P^{\prime}$ is central in $N$, and in particular, $N^{\prime}=P^{\prime}$ is abelian.

We have seen that each linear character $\lambda$ of $N$ extends to $P$ and since $P / N$ is abelian, it follows that $\operatorname{cd}(G \mid \lambda)$ consists only of numbers that are coprime to $p$. A character of degree $b$ in $\operatorname{cd}(G \mid N)$, therefore, lies over nonlinear irreducible characters of $N$, and hence it lies over a nonprincipal linear character of $N^{\prime}$. (Recall that $N^{\prime}$ is abelian.) Since $r$ does not divide $b$, it follows that $N^{\prime}$ has nonprincipal $R$-invariant linear characters, and thus $\left[N^{\prime}, R\right]<N^{\prime}$ for every choice of $R \in \operatorname{Syl}_{r}(G)$.

Recall that $R$ centralizes $P / N$, and thus $P$ normalizes $N R$. Also, $N^{\prime}$ is central in $N$, and thus $\left[N^{\prime}, R\right]=\left[N^{\prime}, N R\right]$ is normalized by $P$. Then $P$ acts on the nontrivial $p$-group $N^{\prime} /\left[N^{\prime}, R\right]$ and we can choose a nonprincipal $P R$-invariant linear character $\sigma$ of $N^{\prime}$. Observe that $\sigma$ is also $Q$-invariant since we have seen that $Q$ centralizes $N^{\prime}$, where $Q$ is a Hall $\pi$-subgroup of $G$. Since $P Q R=G$, we conclude that $\sigma$ is actually $G$-invariant.

Let $X$ be a $p$-complement in $G$ and note that since $\sigma$ is $X$-invariant, there exists a $X$-invariant character in $\operatorname{Irr}(P \mid \sigma)$. (See Theorem 13.28 of 3].) It follows that $\operatorname{cd}(G \mid \sigma)$ contains a power of $p$, and it is no loss to assume that $b \in \operatorname{cd}(G \mid \sigma)$.

Since $G / P$ has more than one Sylow $r$-subgroup, we see that $\operatorname{cd}(G / P)$ contains a multiple of $r$. Also, an irreducible character of $G$ of degree $b$ restricts irreducibly to $P$, and so by Gallagher's theorem, $\operatorname{cd}(G \mid \sigma)$ contains a multiple of $b r$. We may assume, therefore, that this degree is $z$, and so if we write $L=\operatorname{ker}(\sigma)$, we have $z, b \in \operatorname{cd}((G / L) \mid(N / L))$. We claim that this set also contains $a, x$ and $y$. This is because an irreducible character of $G$ with one of these degrees has $p^{\prime}$-degree, and thus has linear constituents upon restriction to $N$. Therefore $L \subseteq N^{\prime}$ is contained in the kernel of any such character. Thus $\operatorname{cd}((G / L) \mid(N / L))$ contains $a, x$ and $y$, and it follows that $a$ and $b$ are in the same connected component of the subgraph $\mathcal{G}((G / L) \mid(N / L))$ of $\mathcal{G}(G \mid N)$, and so their distance in this subgraph is 4 . By the minimality of $|G|$, we see that $L=1$, and thus $\sigma$ is a faithful linear character of $N^{\prime}$, which, therefore, is cyclic. Since $\sigma$ is $G$-invariant, and it follows that $N^{\prime} \subseteq \mathbf{Z}(G)$.

Recall that $X$ is a $p$-complement of $G$, and write $Y=\mathbf{O}^{r^{\prime}}(X)$. Then $Y$ centralizes $P / N$ since we know that each Sylow $r$-subgroup of $G$ centralizes $P / N$, and it 
follows that $P$ normalizes $N Y$. Also, $X$ normalizes $N Y$ since it normalizes both $N$ and $Y$, and since $P X=G$, we conclude that $N Y \triangleleft G$. Now $|Y|$ and $|N|$ are coprime, and hence the subgroup $[N, Y]$ is characteristic in $N Y$ and $[N, Y] \triangleleft G$. Furthermore, since $N^{\prime} \subseteq \mathbf{C}_{N}(Y)$, we see that $\mathbf{C}_{N}(Y) \triangleleft N$, and it follows that $\mathbf{C}_{N}(Y)$ is characteristic in $N Y$ and normal in $G$.

We wish to apply Lemma 7.2 to the action of $Y$ on $N$. Recall first that $N^{\prime}$ is cyclic and central in $N$ and that $Y$ acts trivially on $N^{\prime}$. Also, $X \cong G / P$, and thus $X$ has an abelian normal $r$-complement, and so $Y$ has an abelian normal $r$ complement too. We saw that every linear character of $N$ is fixed by some Sylow $r$-subgroup of $G / P$, and it follows that each such linear character is fixed by a Sylow $r$-subgroup of $X$, which, of course, is a Sylow $r$-subgroup of $Y$. Finally, we know that a linear character of $N$ that is fixed by more than one Sylow $r$-subgroup of $G / P$ is fixed by all of these subgroups, and it follows that a linear character of $N$ that is fixed by more than one Sylow $r$-subgroup of $Y$ is fixed by all of the Sylow $r$-subgroups of $Y$. Such a linear character, therefore, is fixed by $\mathbf{O}^{r^{\prime}}(Y)=Y$.

We can now apply Lemma 7.2 to the action of $Y$ on $N$ and we deduce that $N=[N, Y] \times \mathbf{C}_{N}(Y)$. Since both factors are normal in $G$ and the diameter of $\mathcal{G}(G \mid N)$ exceeds 3 , it follows by Theorem 2.4 that one of the factors must be trivial. We know, however, that $1<N^{\prime} \subseteq \mathbf{C}_{N}(Y)$, and we conclude that $[N, Y]=1$, and thus $Y \triangleleft N Y$. Then $Y$ is characteristic in $N Y$, and hence $Y \triangleleft G$. But $X \cong G / P$

contains more than one Sylow $r$-subgroup, and thus $Y$ contains more than one Sylow $r$-subgroup and in particular, $Y$ is nonabelian. Then $Y \cap G^{\prime}>1$, and since $Y \cap N=1$, we see that Theorem 2.6 applies. We deduce that the diameter of $\mathcal{G}(G \mid N)$ is at most 3, and this is our final contradiction.

\section{REFERENCES}

[1] S. Garrison, On groups with a small number of character degrees. Ph.D. Thesis, University of Wisconsin, Madison, 1973.

[2] B. Huppert, Endliche Gruppen I, Springer-Verlag, Berlin-New York, 1967. MR 37:302

[3] I. M. Isaacs, Character Theory of Finite Groups, Dover, New York, 1994. MR 57:417 (review of 1976 original edition)

[4] I. M. Isaacs and G. Knutson, Irreducible character degrees and normal subgroups, J. of Algebra 199 (1998) 302-326. MR 98m:20013

[5] M. L. Lewis, Solvable groups whose degree graphs have two connected components, J. of Group Theory 4 (2001) 255-275. MR 2002g:20015

[6] M. L. Lewis, Bounding Fitting heights of character degree graphs, J. of Algebra 242 (2001) 810-818. MR 2003d:20010

[7] O. Manz, Degree problems II. $\pi$-separable character degrees, Comm. in Algebra 13 (1985) 2421-2431. MR 86m:20009

[8] O. Manz and T. R. Wolf, Representations of Solvable Groups, Cambridge University Press, Cambridge, 1993. MR 95c:20013

[9] J. McVey, Bounding graph diameters of solvable groups, J. of Algebra (Submitted).

[10] P. P. Pálfy, On the character degree graph of solvable groups II. Disconnected graphs, Studia Sci. Math. Hungarica 38 (2001) 339-355. MR 2002m:20012

[11] T. Yuster, Orbit sizes under automorphism actions in finite groups. J. of Algebra 82 (1983) 342-352. MR 84m:20013

[12] J. Zhang, A note on character degrees of finite solvable groups, Comm. in Algegra 28 (2000) 4249-4248. MR 2001f:20020

Department of Mathematics, University of Wisconsin, 480 Lincoln Dr., Madison WisCONSIN 53706

E-mail address: isaacs@math.wisc.edu 